\title{
Stable iPSC-derived NKX2-1+ Lung Bud Tip Progenitor Organoids Give Rise to Airway and Alveolar Cell Types
}

Renee F.C. Hein ${ }^{1, *}$, Ansley S. Conchola ${ }^{2, *}$, Alexis Fine ${ }^{3}$, Zhiwei Xiao ${ }^{3}$, Tristan Frum ${ }^{3}$, Charlie J. Childs ${ }^{1}$, Yu-Hwai Tsai ${ }^{3}$, Emily M. Holloway ${ }^{1}$, Sha Huang ${ }^{3}$, John Mahoney ${ }^{5}$, Jason R. Spence ${ }^{1-4, \#}$

${ }^{1}$ Department of Cell and Developmental Biology, University of Michigan Medical School, Ann Arbor, MI ${ }^{2}$ Program in Cell and Molecular Biology, University of Michigan Medical School, Ann Arbor, MI

${ }^{3}$ Department of Internal Medicine, University of Michigan Medical School, Ann Arbor, MI

${ }^{4}$ Department of Biomedical Engineering Medicine, University of Michigan Medical School, Ann Arbor, MI ${ }^{5}$ Therapeutics Lab, Cystic Fibrosis Foundation, Lexington, MA,

*These authors contributed equally

\#Correspondence: spencejr@umich.edu

Keywords: lung organoid, bud tip progenitor, human pluripotent stem cell

\section{SUMMARY STATEMENT}

iPSC-derived lung bud tip progenitors emerge in organoid culture, can be isolated and expanded, are transcriptionally similar to primary bud tip progenitors, and can differentiate into airway or alveolar organoids.

\section{ABSTRACT}

Bud tip progenitors (BTPs) in the developing lung give rise to all epithelial cell types found in the airways and alveoli. The current work aimed to develop an iPSC organoid model enriched with stable NKX2-1 ${ }^{+}$ BTP-like cells. Building on prior work, we optimized a directed differentiation paradigm to generate spheroids with robust NKX2-1 expression. Spheroids were expanded into organoids that possessed NKX2-1 ${ }^{+} / \mathrm{CPM}^{+}$BTP-like cells, which increased in number over time. Single cell RNA-sequencing analysis revealed a high degree of transcriptional similarity between induced BTPs (iBTPs) and in vivo BTPs. Using FACS, iBTPs can be purified and expanded as induced bud tip organoids (BBTO), which maintain an enriched population of bud tip progenitors. When iBTOs are directed to differentiate into airway or alveolar cell types using well-established methods, they give rise to organoids composed of organized airway or alveolar epithelium, respectively. Collectively, iBTOs are transcriptionally and functionally similar to in vivo BTPs, providing an important model to study human lung development and differentiation.

\section{INTRODUCTION}

Advances in directed differentiation methods have led to the development of numerous embryonic or induced pluripotent stem cell (iPSC)-derived cell or organoid models of the airway and alveoli, which have 
enhanced our ability to model human lung development and disease (Wang et al., 2007; Mou et al., 2012; Gotoh et al., 2014; Huang et al., 2014; Dye et al., 2015, 2016; Konishi et al., 2016; Chen et al., 2017; McCauley et al., 2017; Yamamoto et al., 2017, 2020; Hawkins et al., 2017; Miller et al., 2018, 2019; Tamò et al., 2018; de Carvalho et al., 2019; Jacob et al., 2019; Leibel et al., 2020). Alveolar and airway cell types in mice and humans are derived from a common, developmentally transient progenitor population, called bud tip progenitors, which reside at the tips of the branching tree-like network of epithelial tubes that make up the lung epithelium (Serra, Pelton and Moses, 1994; Perl et al., 2005; Abler, Mansour and Sun, 2008; Goss et al., 2009; Rawlins et al., 2009; Rockich et al., 2013; Alanis et al., 2014; Nikolić et al., 2017; Miller et al., 2018).

Bud tip progenitors obtained from the human fetal lung and grown as organoids serve as a useful tool to study the mechanisms responsible for bud tip progenitor cell maintenance and differentiation into airway and alveolar cell types (Nikolić et al., 2017; Miller et al., 2018, 2020; Conway et al., 2020; Sun, Evans and Rawlins, 2020). Despite this progress, organoids derived from fetal tissue are not broadly accessible to the research community and are associated with ethical and regulatory challenges, emphasizing the importance of iPSC-derived lung models. While we and others have made some progress in developing bud tip progenitor cell-like models from iPSCs (Chen et al., 2017; Miller et al., 2018), new technologies such as single cell RNA sequencing (scRNA-seq) and single cell lineage tracing have highlighted offtarget cell types and unexpected plasticity in iPSC-derived cultures, alongside unexpected plasticity, where cells appear committed to a specific cell type or cell lineage but change fate (Little et al., 2019; Hurley et al., 2020). This concept of cellular plasticity within the lung has also been demonstrated in vivo, where hyperactive WNT signaling in lung progenitors was shown to cause an emergence of intestinal cells in transgenic mouse embryos (Okubo and Hogan, 2004). Therefore, a current challenge in the field, addressed in the current work, is to develop a long-lived and transcriptionally stable bud tip progenitor-like model from iPSCs.

Single cell RNA sequencing technologies have also made it possible to benchmark iPSC-derived cultures against primary tissue to compare transcriptional similarity and to accurately catalogue the diversity of ontarget or off-target cell types observed in vitro (Hawkins et al., 2017, 2021; McCauley et al., 2018; Holloway et al., 2020; Hor et al., 2020; Yu et al., 2020). Since iPSC-derived cultures are known to be plastic, and the fact that iPSC differentiation is not $100 \%$ efficient, benchmarking has become an important step towards understanding the full complement of cells present in a culture. The current manuscript therefore also sought to benchmark iPSC-derived bud tip progenitor organoids (iBTOs) to interrogate the diversity of cell types in culture and similarity to bud tip progenitor organoids from the fetal lung. 
Here, we optimized an iPSC directed differentiation paradigm to generate self-organizing 3D spheroids with robust NKX2-1 expression. Expansion of NKX2-1 ${ }^{+}$cells in bud tip progenitor media over $3-17$ weeks gives rise to heterogenous organoids that contain NKX2-1+ bud tip progenitor-like cells coexpressing markers of human bud tip progenitors, including SOX9, SOX2 and the cell surface marker CPM (Yamamoto et al., 2020). Using an NKX2-1 reporter iPSC line along with CPM to quantitatively assess cultures via flow cytometry, we observed that bud tip progenitor-like cells expanded over subsequent weeks in culture. FACS isolation and further culture allowed for the expansion of NKX2$1^{+} / \mathrm{CPM}^{+}$cells as bud tip progenitor-like organoids (iBTOs) that maintained $\sim 85 \% \mathrm{NKX} 2-1^{+} / \mathrm{CPM}^{+}$cells for at least 8 weeks. scRNA-seq analysis of bud tip progenitor cells from unsorted organoids or following FACS-enrichment revealed a high degree of transcriptional similarity to fetal-derived bud tip organoids as well as a shared transcriptional signature with in vivo bud tip progenitors. In addition, scRNA-seq from iBTOs that have spent less ( 3 weeks) or more (10 weeks) time in culture suggest that induced bud tip progenitors become more transcriptionally similar to native bud tip progenitors as they age. Finally, we used well-established methods to direct differentiation of iBTOs into organoids composed of airway epithelium (including basal, secretory, ciliated, goblet, and neuroendocrine cells) or alveolar type II (AT2) organoids (Jacob et al., 2017; Miller et al., 2020). Collectively, this study describes a robust method to generate stable bud tip progenitor-like cells from iPSCs that closely resemble organoids derived from primary tissue. This model can be readily used to study lung development and illustrates a proof-ofconcept for cellular engineering and cell therapy.

\section{RESULTS}

\section{Lung Spheroids are Optimized for NXK2.1 Expression but Remain Heterogenous}

NKX2-1 is the earliest marker during lung epithelial specification (Lazzaro et al., 1991) and loss of NKX21 leads to lung agenesis (Minoo et al., 1995, 1999; Little et al., 2019; Kuwahara et al., 2020). We therefore sought to build on a previously-published method to generate iPSC-derived foregut spheroids (Dye et al., 2015) by optimizing endoderm induction efficiency, foregut spheroid development, and finally to improve conditions that induced robust NKX2-1 expression.

We began by testing conditions to improve definitive endoderm (DE) differentiation efficiency and reproducibility. DE induction of varying efficiencies is achieved by using Activin A (ACTA) ligand (Kubo et al., 2004; D'Amour et al., 2006; Spence et al., 2011) with WNT and BMP signaling playing a synergistic role during the initial stages DE specification (Gadue et al., 2006; Green et al., 2011; Loh et al., 2014; Matsuno et al., 2016; Rankin et al., 2018; Heemskerk et al., 2019). Therefore, we tested combinations of ACTA alongside the small molecule WNT activator CHIR99021 (CHIR) or BMP4 on the first day of a three-day ACTA differentiation culture (Fig. S1A,B). Using flow cytometry with a SOX17-tdTomato and SOX2-mCITRINE hESC reporter line (Martyn et al., 2018) to quantitate cell composition, we observed 
that ACTA alone induced $48.41 \%$ SOX $17^{+}$cells, while the addition of CHIR or BMP both enhanced DE differentiation, leading to $96.48 \%$ SOX $17^{+}$cells or $86.83 \%$ SOX $17^{+}$cells respectively. Addition of both CHIR and BMP4 led to $71.03 \%$ SOX $17^{+}$induction (Fig. S1A). DE cultures from an additional cell line were co-stained with SOX17 and FOXA2 to confirm definitive endoderm cell identity (Fig. S1B). We observed that near-pure SOX $17^{+}$cultures obtained via ACTA/CHIR failed to give rise to self-organizing $3 \mathrm{D}$ spheroids (data not shown), consistent with published data showing that self-organizing foregut and hindgut organoids consist of both epithelium and mesenchymal lineages (Spence et al., 2011; Dye et al., 2015, 2016). Therefore, ACTA/BMP4 was used for subsequent experiments.

Following DE specification, monolayers were directed into 3D foregut endoderm spheroids by combining a method that efficiently induces ventral foregut endoderm competent to be specified as lung (Rankin et al., 2016) alongside methods that induce 3D self-organization (Spence et al., 2011; Dye et al., 2015). This included BMP inhibition via NOGGIN (NOG) for 3 days plus all trans retinoic acid (ATRA) on the last day as well as FGF4 and CHIR, which are required for 3D spheroid formation, for all 3 days (Fig. S1C,D). After 3 days, spheroids were collected and suspended in Matrigel and treated for 3 additional days with low-BMP4 as well as WNT3A and RSPO1, which together stimulate WNT signaling (Fig. S1E) (Shu et al., 2005; Goss et al., 2009; Harris-Johnson et al., 2009; Domyan et al., 2011; Jacobs, Ku and Que, 2012; Miller et al., 2012; Serra et al., 2017). WNT activation through RSPO/WNT or CHIR resulted in comparable expression levels of foregut and hindgut markers (Fig. S1E); however, CHIR is a GSK3 $\beta$ inhibitor and can have non-WNT mediated effects, so RSPO/WNT were used as more specific activators of WNT signaling.

At the end of the 9-day directed differentiation (Fig. 1A), spheroids were analyzed for NKX2-1 expression via qRT-PCR (on day 10) (Fig. 1B). When directly compared to spheroids generated using previously published foregut/lung spheroid protocols (Dye et al., 2015; Miller et al., 2019), NKX2-1-optimized foregut spheroids express approximately 100 -fold more NKX2-1. As controls, we included undifferentiated iPSCs and hindgut spheroids, both of which had very low NKX2-1, and RNA from fetal lung was used as a positive control. Using an NKX2-1-EGFP reporter cell line, EGFP was not detected at day 7, whereas lowlevel ubiquitous expression with scattered NKX2-1 ${ }^{\mathrm{HI}}$ cells could be detected starting on day 10 , and expression was localized to specific regions by day 14 (Fig. 1C). Whole mount immunofluorescence (IF) on day 10 spheroids correlated with reporter expression, with individual cells expressing high levels of NKX2-1 protein (Fig. 1D).

When day 10 spheroids were analyzed by scRNA-seq, NKX2-1 was also observed in a subset of cells (Fig. 1E,F), revealing heterogeneity within the foregut spheroids and confirming the findings by EGFP reporter expression. Spheroids included two clusters (Clusters 1 and 2) of lung-fated ventral foregut endoderm-like cells expressing NKX2-1, FOXA2, FOXP1, FOXP4 and HHEX (Bogue et al., 1998; Shu et al., 2007; Spence et al., 2009; Kearns et al., 2013; Davenport et al., 2016; Li et al., 2016). Clusters and 
cells were largely negative for other foregut lineage markers including TP63, TBX1, PDX1, PAX8 and $A L B$; however, there was a clear population of cells that express FOXA2, FOXA3, SOX17 and CDX2 (Cluster 3), which we refer to as "hindgut-primed" endoderm. Additionally, we observed a cluster of CDX2 $/$ HAND $1^{+} / / S L 1^{+} / B M P 4^{+} / F O X F 1^{+} / L E F 1^{+}$(Cluster 4) foregut mesoderm-like cells (Han et al., 2020) and a small population of cells (Cluster 5 ) expressing markers indicative of primordial germ cells, including POUF1, NANOG, T, SOX17, NANOS3 and TFAP2C (Davenport et al., 2016; Jo et al., 2021) (Fig. 1F, S1D). Comparing optimized foregut spheroids to previously published methods (Dye et al., 2015; Miller et al., 2019) by qRT-PCR, we observed that the hindgut endoderm marker CDX2 is not statistically different but the posterior foregut endoderm marker SOX2 (Que et al., 2007) is reduced with the optimized method (Fig. S1F). Taken together, our data shows that optimized foregut spheroids have much higher levels of NKX2-1 when compared to prior methods, but they are still heterogeneous with distinct populations of lung-fated cells and hindgut/intestine-fated cells.

\section{iPSC-derived Bud Tip Progenitors Emerge Over Time}

Once NKX2-1+ spheroids were formed, we asked how efficiently these spheroids would give rise to BTPlike cells as spheroids expanded into larger organoid structures. Our previous studies have shown that "3 Factor (3F) media" possessing FGF7, CHIR99021 and ATRA expands primary human fetal bud tip progenitors and $\mathrm{SOX}^{+} / \mathrm{SOX} 9^{+}$iPSC-derived BTP-like cells; however, efficiency and culture over prolonged periods of time were not assessed (Miller et al., 2018). After inducing NKX2-1-optimized spheroids on day 10, media was switched to 3F BTP media (Fig. 2A). Spheroids were maintained in 3F for several weeks, where they expanded into complex, branching structures termed "lung progenitor organoids (LPOs)," similar to what we have observed previously (Miller et al., 2018) (Fig. 2B). LPOs were passaged every $2-4$ weeks as either intact organoids with minimum fragmentation (whole-passaged) or were sheared by being drawn through a hypodermic needle or pipette, which is a standard method for passaging primary BTP organoids (Miller et al., 2018, 2020; Hein et al., 2021). Using an NKX2-1-EGFP reporter iPSC line, to compare retention of lung identity after various passaging methods, only wholepassaged organoids maintained robust EGFP+ reporter expression (Fig. 2C). Sheared LPOs recovered well and proliferated faster than whole-passaged LPOs (data not shown); however, fragmenting organoids ultimately led to a loss of NKX2-1-EGFP+ cells (Fig. 2C, quantified in Fig. 2F). Based on the maintenance of NKX2-1 expression, we therefore chose to use whole-passaged LPOs for our remaining experiments.

We evaluated the presence of BTP-like cells within whole-passaged LPOs by IF of paraffin sections and whole mount (Fig. 2D, S2A). LPOs possessed regions of NKX2-1-expressing cells, which co-expressed the BTP markers CPM, SOX9 and SOX2 (Nikolić et al., 2017; Miller et al., 2018; Yamamoto et al., 2020) (Fig. 2D, S2A). LPOs also contained sub-regions of distinct NKX2-1\%CDX2+ cells, with many expressing MUC2, indicative of goblet-like cells, and smaller regions of NKX2-1\%CDX2 cells (Fig. 2E, S2B). 
We quantitatively assessed EGFP $/ \mathrm{CPM}^{+}$cells in LPOs grown for $3-17$ weeks in $3 \mathrm{~F}$ media using fluorescence activated cell sorting (FACS) (Fig. 2F, S2C,D). We observed an increase in $\mathrm{EGFP}^{+} / \mathrm{CPM}^{+}$ cells in culture over time (Fig. 2F, purple bars). At 3 weeks, $12 \%$ of cells expressed CPM and EGFP, in contrast to 17 weeks, where $62 \%$ of cells in culture were $\mathrm{EGFP}^{+} / \mathrm{CPM}^{+}$(Fig. $\left.2 \mathrm{~F}\right)$. A small portion $(<10 \%)$ of cells were singly positive for EGFP (green) or CPM (blue) at any timepoint, and a population of doublenegative cells were observed at all times, suggesting that some heterogeneity is maintained in these organoids. We interrogated LPOs derived from two additional non-reporter iPSC lines, using only CPM to quantify BTP-like cells (Fig. S2C). We observed a similar increase of BTP-like cells in culture over time with $\sim 10 \% \mathrm{CPM}^{+}$cells at 3.5 weeks and nearly $50 \% \mathrm{CPM}^{+}$cells by 17 weeks (Fig. S2C). Lastly, organoids derived from previously published lung organoid protocols (Dye et al., 2015; Miller et al., 2019) contained approximately $3-15 \% \mathrm{CPM}^{+}$cells at any timepoint examined (Fig. S2C, right panel). The increase in $\mathrm{CPM}^{+}$cells over time suggests that optimized culture conditions promote the emergence and selection of iBTPs.

To further interrogate the heterogeneity and complexity of the LPOs, we performed SCRNA-seq on wholepassaged LPOs at 3, 6, and 10 weeks (Fig. 2G, S2E-G, S3). We identified one cluster expressing robust levels of NKX2-1 and BTP genes (Cluster 2), several clusters expressing hindgut markers (Clusters 0,5 , 6 ), a mesenchymal cluster (Cluster 3 ), a neuroendocrine-like cluster (Cluster 4 ) and a cluster of unknown/uncommitted cells (Cluster 1) (Fig. 2G, S2E-G). The proportion of cells in the BTP cluster (Cluster 2 ) increased over time while mesenchyme (Cluster 3 ) was depleted over time and hindgut cells (Clusters $0,5,6$ ) were persistent (Fig. S2F). Together this data supports FACS data suggesting that iBTPs continue to expand within LPOs over time and identifies contaminating lineages that persist.

\section{iPSC-derived Bud Tip Progenitors can be Isolated, Expanded and Maintained Long-term}

Although LPOs at every time point contain a significant population of induced BTPs (iBTPs), given the large population and persistence of hindgut lineages, we aimed to isolate $\mathrm{NKX} 2-1-\mathrm{EGFP}^{+} / \mathrm{CPM}^{+}$cells to generate higher purity induced bud tip organoid (BBT) cultures. IBTPs were isolated via FACS with CPM and NKX2-1-EGFP (or CPM only for non-reporter cell lines) and were replated in Matrigel at 8,000 cells/ $\mu \mathrm{L}$ in $3 \mathrm{~F}$ media to form iBTOs (Fig. 3A,B). The NKX2-1-EGFP reporter line showed uniform expression of NKX2-1-EGFP in iBTOs (Fig. 3B) and IF of paraffin sections showed iBTOs contain a nearhomogenous population of cells expressing BTP markers CPM, SOX9 and SOX2 (Fig. 3C, S4). Interestingly, the age of LPOs at the time of sorting was a critical determinant of the ability of iBTOs to maintain NKX2-1 and CPM expression. For example, iBTOs generated from 3-week LPOs maintain $4.05 \%$ NKX2-1-EGFP ${ }^{+}$CPM $^{+}$cells when resorted 7 weeks later. In contrast, iBTOs from >8-week LPOs maintain $\sim 83 \%$ NKX2-1-EGFP ${ }^{+} / \mathrm{CPM}^{+}$cells when resorted 8 weeks later (Fig. 3D). This suggests that 
iBTPs undergo increasing commitment to a bud tip progenitor identity as they are maintained in $3 \mathrm{~F}$ at the LPO stage.

\section{iPSC-derived Bud Tip Progenitors are Transcriptionally Similar to Human Fetal Bud Tip Progenitor Cultures}

To further interrogate iBTO cellular composition, and to directly compare cells within iBTOs to primary human fetal bud tip progenitors and organoids derived from fetal bud tips (Miller et al., 2018), we performed scRNA-seq on iBTOs derived from 4- and 10-week LPOs. iBTOs were expanded for 4 weeks in culture before scRNA-seq was carried out. Integrated analysis of both data sets resulted in 4 epithelial clusters, (Fig. 3E, S4B). Cluster 4 expressed hindgut/intestinal markers (i.e. CDX2), which could also be identified by immunofluorescence (Fig. S4A) and represented a small fraction of cells that are predominantly derived from the 4-week sample (Fig. 3G). The remaining 3 clusters (Clusters 1, 2, 3) express NKX2-1 as well as BTP markers (SOX9, CPM, ETV5, TESC, FGF20, SOX11, HMGB2, NPC2, LGR5, ETV4) (Miller et al., 2018, 2020; Hein et al., 2021); however, they also expressed proliferation genes at varying levels, suggesting there is some heterogeneity within iBTPs that is likely driven by the expression of proliferation genes (Fig. $3 \mathrm{H}$ ).

To benchmark transcriptional similarities iBTPs to in vivo BTPs, we re-analyzed published scRNA-seq data from the human fetal lung (Miller et al., 2020; Hein et al., 2021) and identified a panel of the top 93 enriched genes expressed in in vivo BTPs relative to all other epithelial cell types in the developing lung (See 'Methods'). We used this gene list as a reference to assign an "in vivo bud tip progenitor cell score" to each individual cell from the LPOs and iBTOs using published computational methods (Holloway et al., 2020; Hein et al., 2021). For the purposes of this comparison, both whole LPOs and extracted NKX2-1 cells from the LPO data sets were included in the analysis. As additional control comparisons, we analyzed published data and included scores for BTOs derived from fetal lung, in vivo basal cells and in vivo neuroendocrine cells (Miller et al., 2018, 2020; Hein et al., 2021) (Fig. 3I). As expected, in vivo BTPs had the highest score (median score $=0.80$ ) while in vivo basal cells, in vivo neuroendocrine cells and whole LPOs had the lowest scores $(-0.01,0.05,0.03$ respectively). A noteworthy observation is that human fetal BTOs had a mean BTP score of 0.31 , which was much lower than the in vivo BTP score of 0.80 , indicating that the in vitro culture conditions significantly influence gene expression, as has been observed previously (Miller et al., 2020). Relative to fetal-derived BTOs, both NKX2-1+ cells from LPOs and iBTOs show high transcriptional similarity; their scores improve with longer time in culture at the LPO stage ( 0.21 to 0.27 for $N K X 2-1^{+}$cells from LPOs; 0.21 to 0.30 for iBTOs) (Fig. 3I). To provide additional confidence in this comparison method, we generated a similar in vivo basal cell score and in vivo neuroendocrine cell score, and observed that the expected populations (i.e. in vivo basal cells and in vivo neuroendocrine cells, respectively) scored the highest (Fig. S4C,D). Taken together, our data supports 
the conclusion that iBTOs represent a stable bud tip progenitor cell that can be maintained in vitro and that share a high degree of transcriptional similarity to fetal bud tip progenitors.

\section{iBTOs Can Give Rise to Airway or Alveolar Fates}

Given that BTPs in the developing lung give rise to alveolar and airway fates, we hypothesized that iBTOs could be guided into airway and alveolar lineages. To test this possibility, we used methods previously developed to efficiently induce lung progenitors into airway (Miller et al., 2020) and alveolar lineages (Jacob et al., 2017) (Fig. 4A). Airway induction involved 3 days of dual-SMAD activation (DSA) followed by 18 days of dual-SMAD inhibition (DSI) and resulted in condensed structures that maintained NKX2-1EGFP expression and expressed mCherry driven by the TP63 promoter (TP63-mCherry) (Fig. 4B, left column). By immunofluorescence, we observed that TP63 expression was highly induced after 3 days of DSA, as expected, and after 18 days of DSI, $\mathrm{TP}^{+} 3^{+}$cells organized around the outside of the organoids (Fig. 4C, S4B).

We performed airway induction on unsorted LPOs as well, hypothesizing that airway differentiation may select for expansion of lung lineages within the LPO, while suppressing non-lung lineages.TP63$\mathrm{mCherry}^{+}$cells were quantified using flow cytometry on both LPOs and iBTOs following the 21-day DSA/DSI protocol or untreated controls (Fig. 4D). Untreated LPOs contained 0.36\% NKX2-1EGFP $^{+} /$TP63-mCherry $^{+}$cells while untreated iBTOs contained $0.76 \%$. Only upon treatment with the 21 day DSA/DSI protocol were significant numbers of EGFP ${ }^{+} / \mathrm{mCherry}^{+}$cells detected $(9 \%$ in LPOs, $16 \%$ in iBTOs) (Fig. 4D, S4B). DSA/DSI-treated iBTOs also maintained a large portion (70\%) of NKX2-1EGFP $^{+} /$TP63-mCherry cells, which represented the spectrum of differentiated airway epithelial cell types identified by immunofluorescence, including multiciliated, neuroendocrine, goblet and secretory cells (Fig. 4D,E). Airway organoids contained AcTUB ${ }^{+} / M^{\prime} C_{16}{ }^{+}$ciliated cells (Carraro et al., 2021) (Fig. 4E), and $\mathrm{SYN}^{+}$cells were double positive for ASCL1 (early neuroendocrine marker) or CHGA (late neuroendocrine marker) (Fig. 4E). Treated LPOs contained a small proportion of cells only expressing TP63-mCherry (6\%) (Fig. 4D, S4B) and majority were negative for both markers (76\%), suggesting non-lung lineages continue to expand in these conditions

Alveolar induction consisted of 7 days of treatment with $\mathrm{CK}+\mathrm{DCl}$ as has been previously described with the exception of an alternate composition of the base media (see 'Methods') (Jacob et al., 2017). Treatment of $\mathrm{iBTOs}$ with $\mathrm{CK}+\mathrm{DCl}$ resulted in expanded budded structures that maintained NXK2.1EGFP expression (Fig. 4B, right column). Alveolar type II markers SFTPB, SFTPC and ABCA3 were low to undetectable in iBTOs but were highly expressed upon treatment with $\mathrm{CK}+\mathrm{DCl}$ as determined by qRT-PCR and co-expressed within the same cells as shown by immunofluorescence for (pro-)SFTPC, SFTPC, SFTPB and NKX2-1 (Fig. 4F,G). The lung-specific epithelial marker NKX2-1 was robust in both airway and alveolar organoids while expression of the intestinal epithelial marker CDX2 was not regularly 
detected (Fig. 4D-G, S4A). Taken together, differentiation of iBTOs into airway and alveolar cell types with minimal contaminating non-lung cell types supports that iBTOs have similar developmental potential to in vivo bud tip progenitors.

\section{DISCUSSION}

With the current work, we demonstrate an optimized in vitro model system for differentiating iPSCs into an NKX2-1-expressing bud tip progenitor lineage, which we show can give rise to both airway and alveolar cell types. Several groups, including ours (Dye et al., 2015, 2016; Miller et al., 2018, 2019), have previously characterized protocols that yield lung-like cell types from iPSCs (Wang et al., 2007; Mou et al., 2012; Gotoh et al., 2014; Huang et al., 2014; Konishi et al., 2016; Abler et al., 2017; Chen et al., 2017; Hawkins et al., 2017; McCauley et al., 2017; Tamò et al., 2018; de Carvalho et al., 2019; Jacob et al., 2019; Yamamoto et al., 2020; Leibel et al., 2020). While many of these protocols capture a transient bud tip progenitor-like stage, they focus primarily on deriving more mature lung epithelial lineages, and the induction and maintenance of NKX2-1+ bud tip progenitor-like cells from iPSCs has not been previously reported. Furthermore, benchmarking of induced cells using scRNA-seq in order to compare cell types to a human reference atlas has only recently become commonplace (Holloway et al., 2020; Hor et al., 2020; Yu et al., 2020; Hawkins et al., 2021). The method reported here expands upon an improved understanding of critical signaling events that regulate bud tip progenitor maintenance in the human lung to better mirror this process in the dish (Conway et al., 2020).

Although we demonstrate that optimizing NKX2-1 expression leads to a 100-fold induction when compared to previously reported methods (Dye et al., 2015; Miller et al., 2019), scRNA-seq and whole mount immunofluorescence analysis of day 10 spheroids revealed that low levels of NKX2-1 is expressed in many cells, while a small number of cells express high levels of NKX2-1, highlighting the heterogeneity that still exists at this early timepoint. We also observed non-lung lineages that co-emerge in spheroids, possibly due to the fact that the same signaling pathways play a role during lineage choice for multiple lineages, and due to the inherent plasticity of newly committed cells. We observed that non-lung lineages, and particularly gut lineages, persisted over time in culture. Based on this observation, there is an opportunity to further refine the signaling pathways that are manipulated to expand and maintain NKX2-1 $1^{+}$ lung lineages; however, this may prove challenging given the redundant use of some pathways (i.e. WNT) to maintain stem/progenitor cells from both lineages (Pinto and Clevers, 2005; Kapoor, Li and Leiter, 2007; Volckaert and De Langhe, 2015; Chin et al., 2016; Nikolić and Rawlins, 2017; Yan et al., 2017; Miller et al., 2018; Rabata et al., 2020; Aros, Pantoja and Gomperts, 2021). We observed that hindgut lineages expand after passaging organoids using the standard approach of mechanically shearing them into small fragments. It is currently unclear if this phenomenon occurs because shearing increases selection for highly proliferative $\mathrm{CDX} 2^{+}$cells or if the mechanical stress results in higher levels of apoptosis in one lineage (i.e. lung) compared to the other. Thus, while we have optimized the current 
methods, to generate a high proportion of NKX2-1+ cells, LPO cultures still exhibit high plasticity that can be triggered through perturbations such as organoid dissociation.

One important observation from our current study is that iBTPs exhibit increased lineage commitment with longer time in culture. Isolating NKX2-1-EGFP ${ }^{+} / \mathrm{CPM}^{+}$cells prior to 3 weeks resulted in cultures replete with gut cells. While follow-up experiments are required to determine the origin of contaminating gut cells, the fact that we still see these cells in iBTOs suggests a fate switch from a lung bud tip progenitor to a hindgut identity. On the other hand, purifying cells at $6-10$ weeks reliably led to the establishment of $\mathrm{NKX} 2-1-\mathrm{EGFP}^{+} / \mathrm{CPM}^{+}$iBTOs that expand and maintain their fate. We attempted airway differentiation on unsorted LPOs, hypothesizing that the process of differentiation itself may select against gut lineages and expand only lung lineages; unfortunately, non-lung lineages persisted. Given this result, sorting remains an important step in establishing iBTO cultures that can be differentiated into airway or alveolar cell types in the absence of non-lung cell types. Finally, the experiments carried out here relied heavily on an NKX21-EGFP reporter; however, our results suggest that this should not limit studies with non-reporter iPSC lines, since we also observed that sorting with CPM alone highly enriched for iBTPs. As we detected some NKX2-1-EGFP-/CPM ${ }^{+}$, identifying additional bud tip progenitor cell surface markers to further improve purity of cultures may be valuable for enhancing purity in non-reporter iPSC cultures.

The utilization of emerging technologies such as scRNA-seq has provided critical insights into the heterogeneity and complexity of human tissues that were once difficult to study (Miller et al., 2020; Yu et al., 2020). New information from human tissue has provided both an atlas against which in vitro model systems can be benchmarked and a roadmap that can be used to infer transcriptional and signaling mechanisms that control cellular transitions. Here, we used scRNA-seq to benchmark in vitro cultures at several stages during differentiation to catalog the induction, emergence, and maintenance of lung-fated cells as they acquire a bud tip progenitor fate. One interesting observation from the benchmarking carried out in this study is a significant shift in the transcriptome when comparing primary in vivo tissue to in vitro grown primary organoids, even when the source of the cells is the same. For example, here, we compared iBTOs to BTOs derived from fetal lung and to in vivo fetal bud tip progenitors. We observed a significant shift when comparing fetal lung bud tip progenitors to primary BTOs, indicating that the in vitro environment significantly changes the transcriptome of the cell, as has been recently reported (Miller et al., 2020; Alysandratos et al., 2022). With this caveat in mind, iBTOs shared a nearly identical transcriptome to primary BTOs, and shared a high degree of similarity to in vivo primary bud tip progenitors. The cell scoring metric used to compare similarity across samples also suggested that iBTOs increased in transcriptional similarity to in vivo bud tip progenitors as they spent more time in culture, supporting the idea that iBTPs undergo continued differentiation towards a bud tip progenitor identity as they are maintained in culture. 
Functionally, iBTOs also behave like bud tip progenitors. Using methods to differentiate lung progenitor cells into airway or alveolar cell types (Jacob et al., 2017; Miller et al., 2020) (Miller et. al., 2020, Jacob et al., 2017), we demonstrated that iBTOs robustly differentiate into airway and alveolar cell types. Taken together, the current work describes a robust iPSC-derived bud tip progenitor model to study human lung epithelial development and differentiation and uses scRNA-seq to benchmark both on- and off-target cell types present in the cultures. Practically, this model presents a novel opportunity to expand the downstream efforts of lung organoid studies, where iBTOs can be shared to reduce the expertise needed for lung cell generation and ultimately facilitate faster turn-around through iBTO banking. Overall, this study enhances the utility of iPSC-derived lung organoids to interrogate lung development and to study disease and regeneration.

\section{MATERIALS AND METHODS}

\section{Tissue processing, Staining, and Quantification}

All sectioned fluorescent images were taken using a Nikon A1 confocal microscope, an Olympus IX83 inverted fluorescence microscope or an Olympus IX71 inverted fluorescence microscope. Whole mount fluorescent images were taken on a Nikon X1 Yokogawa Spinning Disk Microscope. Acquisition parameters were kept consistent for images in the same experiment and all post-image processing was performed equally on all images in the same experiment. Images were assembled in Adobe Photoshop CC 2022.

\section{Tissue Processing}

Tissue was immediately fixed in $10 \%$ Neutral Buffered Formalin (NBF) for $24 \mathrm{~h}$ at room temperature on a rocker. Tissue was then washed $3 x$ in UltraPure DNase/RNase-Free Distilled Water (Thermo Fisher, Cat\#10977015) for 15 minutes each and then dehydrated in an alcohol series of concentrations dehydrated in UltraPure DNase/RNase-Free Distilled Water for $1 \mathrm{~h}$ per solution: $25 \%$ Methanol, $50 \%$ Methanol, $75 \%$ Methanol, 100\% Methanol, 100\% Ethanol, 70\% Ethanol. Dehydrated tissue was then processed into paraffin blocks in an automated tissue processor (Leica ASP300) with 1h solution changes. 4 (FISH) or 7 (IF) $\mu$ m-thick sections were cut from paraffin blocks onto charged glass slides. For FISH, microtome and slides were sprayed with RNase Away (Thermo Fisher, Cat\#700511) prior to sectioning (within one week of performing FISH). Slides were baked for $1 \mathrm{~h}$ in $60^{\circ} \mathrm{C}$ dry oven (within $24 \mathrm{~h}$ of performing $\mathrm{FISH}$ ). Slides were stored at room temperature in a slide box containing a silica desiccator packet and the seams sealed with paraffin.

\section{Immunofluorescence (IF) Protein Staining on Paraffin Sections}

Tissue slides were rehydrated in Histo-Clear II (National Diagnostics, Cat\#HS-202) 2x for 5 minutes each, followed by serial rinses through the following solutions $2 x$ for $2-3$ minutes each: $100 \% \mathrm{EtOH}, 95 \%$ 
$\mathrm{EtOH}, 70 \% \mathrm{EtOH}, 30 \% \mathrm{EtOH}$, and finally in double-distilled water (ddH2O) 2x for 5 minutes each. Antigen retrieval was performed using $1 \times$ Sodium Citrate Buffer (100mM trisodium citrate (Sigma, Cat\#S1804), 0.5\% Tween 20 (Thermo Fisher, Cat\#BP337), pH 6.0), steaming the slides for 20 minutes, followed by cooling and washing quickly $2 \mathrm{x}$ in $\mathrm{ddH} 2 \mathrm{O}$ and $2 \mathrm{x}$ in $1 \mathrm{X}$ PBS. Slides were incubated in a humidified chamber at RT for $1 \mathrm{~h}$ with blocking solution (5\% normal donkey serum (Sigma, Cat\#D9663) in PBS with $0.1 \%$ Tween 20 ). Slides were then incubated in primary antibody diluted in blocking solution at $4^{\circ} \mathrm{C}$ overnight in a humidified chamber. Next, slides were washed 3x in 1X PBS for 5 minutes each and incubated with secondary antibody with DAPI (1ug/mL) diluted in blocking solution for $1 \mathrm{~h}$ at RT in a humidified chamber. Slides were then washed $3 x$ in 1 X PBS for 5 minutes each and mounted with ProLong Gold (Thermo Fisher, Cat\#P36930) and imaged within 2 weeks. Stained slides were stored in the dark at $4{ }^{\circ} \mathrm{C}$. Secondary antibodies, raised in donkey, were purchased from Jackson Immuno and used at a dilution of 1:500.

Fluorescence in situ hybridization (FISH)

The FISH protocol was performed according to the manufacturer's instructions (ACDbio, RNAscope multiplex fluorescent manual) with a 5-minute protease treatment and 15-minute antigen retrieval. For IFco-staining with antibodies, the last step of the FISH protocol was skipped and instead the slides were washed 1x in PBS followed by the IF protocol above.

\section{Whole Mount IF Protein Staining}

All tips and tubes were coated with 1\% BSA in PBS to prevent tissue sticking. 3D cultures (spheroids, LPOs) were dislodged from Matrigel using a P1000 cut tip and transferred to $1.5 \mathrm{~mL}$ Eppendorf tube. $500 \mu \mathrm{L}$ of Cell Recovery Solution (Corning, Cat\#354253) was added to the tube and placed on a rocker at $4^{\circ} \mathrm{C}$ for 45 minutes to completely dissolve Matrigel. Tube was spun at $100 \mathrm{~g}$ for 5 minutes, and solution and remaining Matrigel was then removed. Tissue was fixed in 10\% NBF overnight at RT on a rocker. Tissue then was washed $3 \mathrm{x}$ for $2 \mathrm{hrs}$ with $1 \mathrm{~mL}$ room temperature Organoid Wash Buffer (OWB) $(0.1 \%$ Triton, $0.2 \%$ BSA in $1 \mathrm{XPBS}$ ), at RT on a rocker. Wash times vary (30 minutes -2 hours) depending on tissue size. $1 \mathrm{~mL}$ CUBIC-L (TCI Chemicals Cat\#T3740) was added to the tube and placed on a rocker for $24 \mathrm{hrs}$ at $37^{\circ} \mathrm{C}$. Tissue was then permeabilized for $24 \mathrm{hr}$ at $4^{\circ} \mathrm{C}$ on rocker with $1 \mathrm{~mL}$ permeabilization solution ( $5 \%$ Normal Donkey Serum, $0.5 \%$ Triton in $1 \mathrm{X}$ PBS). After $24 \mathrm{hr}$, permeabilization solution was removed and $500 \mu \mathrm{L}$ primary antibody (diluted in OWB) was added overnight at $4^{\circ} \mathrm{C}$ on a rocker. The next day, tissue was washed $3 \mathrm{x}$ with $1 \mathrm{~mL}$ of OWB, $2 \mathrm{hr}$ each at RT. $500 \mu \mathrm{L}$ of secondary antibody (diluted in OWB) was added and incubated overnight at $4^{\circ} \mathrm{C}$, wrapped in foil. Tissue was washed again $3 \mathrm{x}$ with $1 \mathrm{~mL}$ OWB at RT, first wash for $2 \mathrm{hrs}$, then 30 mins for the remaining washes. Samples were transferred to imaging plate (ThermoFisher Cat\#12-566-70) and then cleared and mounted with 50 $\mu \mathrm{L}$ CUBIC-R (just enough to cover tissue) (TCl Chemicals Cat\#T3741). 
IF and FISH stains were repeated on at least 3 independent differentiations and representative images are show.

\section{Cell Lines \& Culture Conditions}

\section{$\underline{\mathrm{hPSC} \text { Lines and Culture Conditions }}$}

hPSC Lines

LPOs were generated from three human iPSC lines: WTC11 (RRID: CVCL_Y803) was obtained from Bruce Conklin at the University of California San Francisco (Kreitzer et al., 2013), human iPSC line 72.3 was obtained from Cincinnati Children's Hospital Medical Center (McCracken et al., 2014) and iPSC17 WT 7B2, expressing NKX2-1-EGFP and TP63-mCherry, was obtained from the Cystic Fibrosis Foundation (Crane et al., 2015). In addition to iPSC lines, hESC lines were used for definitive endoderm and spheroid optimizations: hESC line $\mathrm{H9}$ (NIH registry no. 0062) was obtained from the WiCell Research Institute and hESC line RUES2-GLR (NIH registry no. 0013), expressing SOX2-mCitrine, BRA-mCerulean and SOX17-tdTomato, was obtained from The Rockefeller University (Martyn et al., 2018). The University of Michigan Human Pluripotent Stem Cell Research Oversight Committee approved all experiments using human embryonic stem cell (hESC) and induced pluripotent stem cell (iPSC) lines. Stem cell lines were routinely karyotyped, and all cell lines are routinely monitored for mycoplasma infection monthly using the MycoAlert Mycoplasma Detection Kit (Lonza, Cat\#LT08-118). Stem cells were maintained as previously described (Spence et al., 2011) and grown in mTeSR Plus media (StemCell Technologies, Cat\#1000276).

\section{NKX2-1-optimized Spheroid Differentiation Protocol}

Generation of definitive endoderm (DE) from hPSCs (differentiation days $1-3$ ) was carried out as previously described with slight modifications (D'Amour et al., 2006; McCracken et al., 2011; Spence et al., 2011; Dye et al., 2015). Briefly, 100ng/mL ActivinA (R\&D Systems, Cat\#338-AC) was added in RPMI 1640 media (Thermo Fisher, Cat\#21875034) with increasing concentrations of HyClone defined FBS (dFBS) (Fisher Scientific, Cat\#SH3007002) on subsequent days ( $0 \%$ day $1,0.2 \%$ day $2,2 \%$ day 3 ). 50ng/mL BMP4 (R\&D Systems, Cat\#314-BP) was added on day 1. Following DE specification, anterior

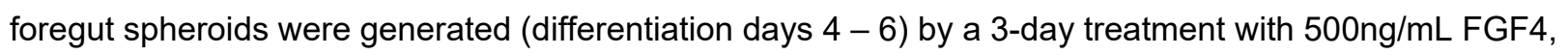
200ng/mL NOGGIN (R\&D Systems, Cat\#6057-NG), 2uM CHIR99021 (APExBIO, Cat\#A3011) and 2uM all trans retinoic acid (Sigma, Cat\#R2625) on the final day. Self-organizing 3D spheroids that had detached from the tissue culture dish were collected with a P200 pipette and were transferred into Matrigel (Corning, Cat\#354234) as previously described (McCracken et al., 2011; Miller et al., 2019). After the Matrigel had solidified, encapsulating spheroids, they were cultured in media that contained $250 \mathrm{ng} / \mathrm{mL}$ WNT3a (R\&D Systems, Cat\#5036-WN), 500ng/mL RSPO1 and 10ng/mL BMP4 for 3 days to induce 
NKX2-1+ cells (differentiation days $7-9$ ). Following day DE specification, RPMI 1640 media $+2 \%$ dFBS was used on all days to dilute growth factors. Fresh media and growth factors were added each day.

\section{Growth and Maintenance of LPOs}

NKX2-1-optimized spheroids were transferred to "3 Factor" (3F) bud tip maintenance media as previously described (Miller et al., 2018, 2019), including 50nM all trans retinoic acid (Sigma, Cat\#R2625), 10ng/mL

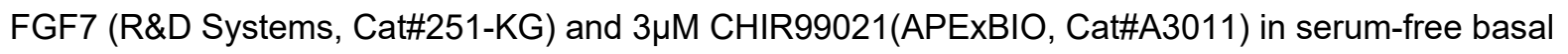
media. Serum-free basal media consists of DMEM/F12 containing HEPES and L-Glutamine (Corning, Cat\#10-092-CV), 100U/mL penicillin-streptomycin (Thermo Fisher, Cat\#15140122), 1x B-27 supplement (Thermo Fisher, Cat\#17504044), 1x N-2 supplement (Thermo Fisher, Cat\#17502048), 0.05\% BSA

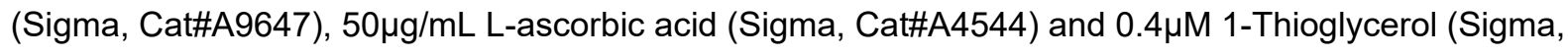
Cat\#M1753). LPOs were grown for 3 weeks, then whole passaged or needle or pipette sheared every 2 4 weeks. Whole passaging was achieved by collecting LPOs into an Eppendorf tube and gently releasing them from Matrigel by a P1000 cut pipette tip. LPOs were spun in a microcentrifuge tube, residual media and Matrigel was removed, then LPOs were re-suspended in Matrigel (Corning, Cat\#354234) with a P1000 cut pipette tip. $\sim 35 \mu \mathrm{L}$ droplets of Matrigel were placed into the center of wells of a 24-well tissue culture plate (Thermo Fisher, Cat\#12565163), and the plate was inverted and placed in an incubator at $37^{\circ} \mathrm{C}$ for 20 minutes. For LPOs passaged by needle or pipette shearing, LPOs were passed through a $27-$ gauge needle or P200 pipette, respectively, and embedded in fresh Matrigel as previously described (Miller et al., 2018, 2019, 2020). LPOs were fed with 3F media every $2-4$ days.

\section{Growth and Maintenance of iBTOs}

iBTs in LPOs grown in 3F media for 3-17 weeks were isolated by FACS (see below). After collection, iBTs were centrifuged at $300 \mathrm{~g}$ for 5 minutes at $4^{\circ} \mathrm{C}$ and media was removed. Matrigel (Corning, Cat\#354234) was added to the cells at a concentration of 8,000 cells $/ \mu \mathrm{L}$ Matrigel and $3-20 \mu \mathrm{L}$ droplets of Matrigel were placed into the center of wells of a 24-well tissue culture plate (Thermo Fisher, Cat\#12565163). The plate was inverted and placed in an incubator at $37^{\circ} \mathrm{C}$ for 20 minutes. iBTPs were fed every $2-4$ days with 3F media and allowed to reform organoids. iBTOs were whole passaged as described above every $2-4$ weeks.

\section{Airway and Alveolar Differentiations}

Airway differentiation was carried out as previously described (Miller et al., 2020). Briefly, iBTOs were exposed to dual-SMAD activation via 100ng/mL BMP4 (R\&D Systems, Cat\#314-BP-050) and 100ng/mL TGFB1 (R\&D Systems, Cat\#240-B-002) in 3F media for 3 days. On the fourth day, iBTOs were exposed to dual-SMAD inactivation via $1 \mu \mathrm{M}$ A8301 (APExBIO, Cat\#3133), 100ng/mL NOGGIN (R\&D Systems,

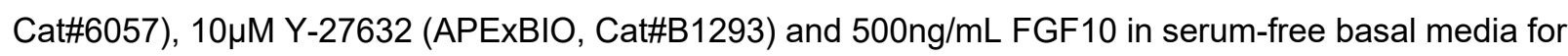
18 days (media changed every $3-4$ days) with whole passaging as necessary. 
Alveolar differentiation was carried out as previously described (Jacob et al., 2017). Briefly, iBTOs were transitioned to alveolar differentiation media for 7 days (media changed on days 3 and 6 ). Alveolar differentiation media consists of a modified serum-free basal media (SFB-VA) with DMEM/F12 containing HEPES and L-Glutamine (Corning, Cat\#10-092-CV) supplemented with 1X N-2 supplement (Thermo Fisher, Cat\#17502048), 1X B-27 supplement without vitamin A (Thermo Fisher, Cat\#12587010), 0.05\%

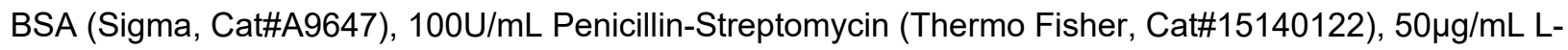

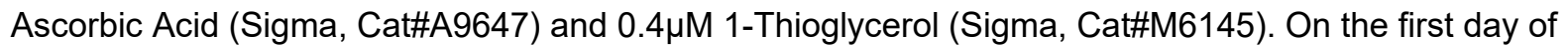
alveolar differentiation, SFB-VA is supplemented with 10ng/mL FGF7 (R\&D Systems, Cat\#251-KG/CF),

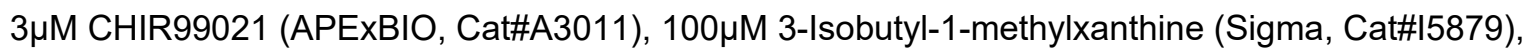

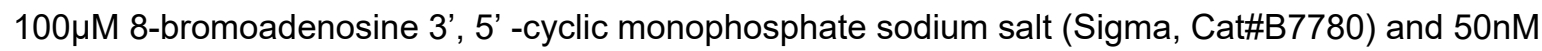
Dexamethasone (Sigma, Cat\#D4802).

\section{$\underline{\text { Culture Media, Growth Factors, Small Molecules }}$}

\section{Tissue Prep for scRNA-seq}

All tubes and pipette tips were pre-washed in 1X HBSS with 1\% BSA to prevent cell adhesion to the plastic. 3D cultures (spheroids, LPOs, iBTOs) were removed from Matrigel using a P1000 pipette tip and vigorously pipetted in a $1.5 \mathrm{~mL}$ Eppendorf tube to remove as much Matrigel as possible. Tissue was centrifuged at $300 \mathrm{~g}$ for 3 minutes at $4^{\circ} \mathrm{C}$, then excess media and Matrigel was removed. Tissue was digested to single cells using $0.5 \mathrm{~mL}$ TrypLE (Invitrogen, Cat\#12605010) and incubated at $37^{\circ} \mathrm{C}$ for 30 minutes, adding mechanical digestion with pipette every 10 minutes. After 30 minutes, trypsinization was quenched with 1X HBSS. Cells were passed through a 40 $\mu \mathrm{m}$ filter (Bel-Art Flowmi, Cat\#136800040), and centrifuged at $300 \mathrm{~g}$ for 3 minutes at $4^{\circ} \mathrm{C}$. Cells were resuspended in $1 \mathrm{~mL} 1 \mathrm{X}$ HBSS and counted using a hemocytometer, centrifuged at $300 \mathrm{~g}$ for 3 minutes at $4^{\circ} \mathrm{C}$ and resuspended to a final concentration of 1,100 cells $/ \mu \mathrm{L}$. If samples were planned for combined submission, cells would be cryopreserved in $\mathrm{XX}$ media and stored in liquid N2. Cells were thawed, washed and sent for sequencing. Approximately 100,000 cells were put on ice and single cell libraries were immediately prepared at the $10 \mathrm{X}$ Chromium at the University of Michigan Sequencing Core with a target of 10,000 cells per sample.

\section{RNA extraction, cDNA, qRT-PCR}

Each analysis includes three biological replicates from three separate differentiation attempts as well as three technical replicates. mRNA was isolated using the MagMAX-96 Total RNA Isolation Kit (Thermo Fisher, Cat\#AM1830) or the PicoPure RNA Isolation Kit (Thermo Fisher, Cat\#KIT0204). RNA quality and yield was measured on a Nanodrop 2000 spectrophotometer just prior to cDNA synthesis. cDNA synthesis was performed using 100ng RNA per sample with the SuperScript VILO cDNA Kit (Thermo Fisher, Cat\#11754250). qRT-PCR was performed on a Step One Plus Real-Time PCR System (Thermo 
Fisher, Cat\#42765592R) using QuantiTect SYBR Green PCR Kit (Qiagen, Cat\#204145). Gene expression as a measure of arbitrary units was calculated relative to GAPDH using the following equation:

$$
2^{\left(\text {GADPH }_{C t}-\text { Gene }_{C t}\right)} \times 10,000
$$

\section{Bioinformatics/scRNAseq}

\section{Overview}

To visualize distinct cell populations within the single-cell RNA sequencing dataset, we employed the recommended workflow outlined by the Seurat 4.0 R package (Hao et al., 2021). This pipeline includes the following steps: filtering cells for quality control, applying the SCTransform technique (Hafemeister and Satija, 2019) in place of traditional log normalization, variable gene selection, and scaling, identifying anchors and integrating if multiple single-cell RNA samples are involved (Stuart et al., 2019), reducing dimensionality with principal component analysis (PCA) and uniform manifold approximation and projection (UMAP) (McInnes, Healy and Melville, 2018; Becht et al., 2019), clustering by either the Louvain algorithm (Blondel et al., 2008) or the Leiden algorithm (Traag, Waltman and van Eck, 2019), and log normalization on RNA assay for final visualization as well as differential gene expression analysis.

\section{Sequencing Data and Processing FASTQ Reads into Gene Expression Matrices}

All single-cell RNA sequencing was performed at the University of Michigan Advanced Genomics Core with an Illumina Novaseq 6000 . The 10X Genomics Cell Ranger pipeline was used to process raw Illumina base calls (BCLs) into gene expression matrices. BCL files were demultiplexed to trim adaptor sequences and unique molecular identifiers (UMIs) from reads. Each sample was then aligned to the human reference genome (hg19) to create a filtered feature bar code matrix that contains only the detectable genes for each sample.

\section{Quality Control}

To ensure quality of the data, all samples were filtered to remove cells expressing too few or too many genes (Fig. 1E-F/Fig. S1G - <500, >8000; Fig. 2G/Fig. S2C-E/Fig. S3A - <500, >7000; Fig. 3E-H/Fig. S4B $-<200,>9500$ ), with too low or too high UMI counts (Fig. 1E-F/Fig. S1G $-<500,>50000$; Fig. 2G/Fig. S2C-E/Fig. S3A - <500, >50000; Fig. 3E-H/Fig. S4B - <200, >50000), or a fraction of mitochondrial genes greater than 0.1. Following the above steps, a total of (Fig. 1E-F/Fig. S1G - 1067 cells, 36601 genes; Fig. 2G/Fig. S2C-E/Fig. S3A - 9133 cells, 36601 genes; Fig. 3E-H/Fig. S4B - 4334 cells, 36602 genes) were kept for downstream analysis and visualization.

\section{SCTransform and Integration}


Seurat's SCTransform method allows efficient pre-processing, normalization, and variance stabilization of molecular count data from scRNA-seq samples. Running this algorithm will reveal a model of technical noise in the scRNA-seq data through "regularized negative binomial regression", whose residuals are returned as the SCTransform-normalized values that can be used for further downstream analysis such as dimension reduction. During the SCTransform process, we also chose to regress out a confounding source of variation - mitochondrial mapping percentage. When dealing with one sample (Fig. 1E-F/Fig. S1G), there's no batch effect. But when multiple samples are present (Fig. 2G/Fig. S2C-E/Fig. S3A/Fig. 3E-H/Fig. S4B), we have noticed certain amount of batch effects when clustering data due to technical artifacts such as timing of data acquisition or differences in dissociation protocol. To mitigate these effects, we chose to follow Seurat's integration workflow due to its optimal efficiency in harmonizing large datasets. After each sample is SCTransform-prepared, a set of anchors, which are mutual nearest neighbors, are identified and filtered. Finally, iterative pairwise integration is performed on the anchor set. After completion of such batch correction, cell clustering should no longer be driven by technical artifacts.

\section{Dimension Reduction and Clustering}

Principal component analysis (PCA) was conducted on the corrected expression matrix followed. Using the top principal components, a neighborhood graph was calculated for the 20 nearest neighbors (Fig. 1E-F/Fig. S1G - 20 principal components; Fig. 2G/Fig. S2C-E/Fig. S3A - 30 principal components; Fig. 3E-H/Fig. S4B - 30 principal components). The UMAP algorithm was then applied for visualization on 2 dimensions. Using the Leiden algorithm, clusters were identified with a resolution of (Fig. 1E-F/Fig. S1G 0.2; Fig. 3E-H/Fig. S4B - 0.2). Using the Louvain algorithm, clusters were identified with a resolution of (Fig. 2G/Fig. S2C-E/Fig. S3A-0.08)

\section{Cluster Annotation}

Using canonically expressed gene markers, each cluster's general cell identity was annotated. Markers utilized include epithelium (EPCAM, KRT18, KRT8, CLDN6), mesenchyme (POSTN, DCN, COL1A2, COL3A1), neuronal (S100B, STMN2, ELAV4, ASCL1), endothelial (ESAM, CDH5, CLDN5, KDR), proliferative (MKI67, TOP2A, CDK1), primordial germ cell (POU5F1, NANOG, TBXT, NANOS3, TFAP2C), and foregut mesoderm (ISL1, HAND1, BMP4, FOXF1, LEF1).

\section{Cell Scoring}

Application of cell scoring strategy is as previously described (Holloway et al., 2020; Hein et al., 2021). Briefly, cells were scored based on expression of a set of $93-153$ marker genes per cell type. Gene lists were compiled by analyzing previously-published data from human fetal lung (Miller et al., 2020; Hein et al., 2021). Clusters were first identified by major cell classes (epithelium, mesenchyme, neuronal, endothelial, immune), then the epithelium was sub-clustered to identify bud tip progenitor, basal and neuroendocrine cell clusters by visualizing canonical marker gene expression for each respective cell 
type. In the case of bud tip progenitor and basal cells, clusters were again sub-clustered to identify the clusters with enriched bud tip progenitor or basal cell marker expression, respectively. The top 50 genes from each bud tip progenitor, basal or neuroendocrine cell clusters were merged to create the gene sets for cell scoring. After obtaining the scaled expression values for the data set, scores for each cell were calculated with the AddModuleScore function of Seurat. Cell scores were visualized by violin plots.

\section{Normalization for Visualization and Differential Gene Expression}

As recommended by Seurat developers, we employed the method of log normalization on the standard RNA assay for graphing dot plots, feature plots, and conducting DGEs. Expression matrix read counts per cell were normalized by the total expression, multiplied by a scale factor of 10000 , and finally logtransformed. For the differential gene expression testing, we only tested features that are first, detected in a minimum fraction of 0.25 in either of the two cell populations, and second, show at least 0.25 -fold difference in log-scale between the two cell populations on average.

\section{Quantification, Statistical Analysis}

Graphs and statistical analysis for RT-qPCR and FACS quantification were performed in GraphPad Prism Software. See Figure legends for the number of replicates used, statistical test performed, and the pvalues used to determine the significance for each analysis.

\section{Fluorescent Activated Cell Sorting (FACS) and Flow Cytometry}

\section{hPSC Flow Cytometry}

$1 \mathrm{~mL}$ accutase (Sigma, Cat\#A6964) was added to each well of hPSC cultures in a 6-well plate and was incubated at $37^{\circ} \mathrm{C}$ for $5-10$ minutes, until cells detached. An equal volume of mTeSR Plus media

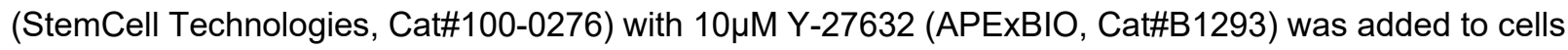
and cells were dissociated mechanically by pipetting with a P1000 pipette $2 \mathrm{x}$, were centrifuged at $300 \mathrm{~g}$ for 5 minutes at $4^{\circ} \mathrm{C}$, excess media was removed, and cells were resuspended in FACS Buffer (2\% BSA, $10 \mu \mathrm{M} Y-27632,100 \mathrm{U} / \mathrm{mL}$ penicillin-streptomycin). Cells were passed through a $70 \mu \mathrm{m}$ cell strainer, precoated with FACS buffer, and centrifuged at $300 \mathrm{~g}$ for 5 minutes at $4^{\circ} \mathrm{C}$. Cells were resuspended in $1 \mathrm{~mL}$ FACS Buffer and transferred to $5 \mathrm{~mL}$ FACS tubes (Corning, Cat\#352063). 0.2 $\mu \mathrm{g} / \mathrm{mL}$ DAPI was added to respective tubes. Flow cytometry was performed using a Bio Rad Ze5\#3 and accompanying software.

\section{$3 D$ Culture Sorting}

3D cultures (LPOs, iBTOs) were removed from Matrigel using a P1000 pipette tip and vigorously pipetted in a $15 \mathrm{~mL}$ conical tube to remove as much Matrigel as possible. Tissue was centrifuged at $300 \mathrm{~g}$ for 3 minutes at $4^{\circ} \mathrm{C}$, then excess media and Matrigel was removed. Tissue was digested to single cells using $2-4 \mathrm{~mL}$ TrypLE (Invitrogen, Cat\#12605010), depending on pellet size, and incubated at $37^{\circ} \mathrm{C}$ for 30 
minutes, adding mechanical digestion with a pipette every 10 minutes. After 15 minutes, DNase I (Qiagen, Cat\#79254) was added to the digestion at $7.5 \mu \mathrm{L} / \mathrm{mL}$ TrypLE. After 30 minutes, trypsinization

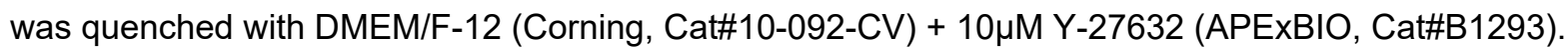
Cells were passed through a $70 \mu \mathrm{m}$ cell strainer, pre-coated with DMEM/F-12 +10 $\mu \mathrm{M} Y-27632$, and centrifuged at $500 \mathrm{~g}$ for 5 minutes at $4^{\circ} \mathrm{C}$. Cells were resuspended in $4 \mathrm{~mL}$ FACS Buffer ( $\%$ BSA, $10 \mu \mathrm{M} \mathrm{Y-}$ 27632, 100U/mL penicillin-streptomycin) and transferred into $5 \mathrm{~mL}$ FACS tubes (Corning, Cat\#352063). Cells were centrifuged again at $300 \mathrm{~g}$ for 3 minutes at $4^{\circ} \mathrm{C}$, then resuspended in $1 \mathrm{~mL}$ FACS buffer and counted. $10^{5}$ cells were placed into new FACS tubes for all controls (no antibody, DAPI only, individual antibodies/fluorophores) and all remaining cells were centrifuged and resuspended in FACS buffer for a concentration of $10^{6}$ cells $/ 100 \mu \mathrm{L}$. Primary antibodies were incubated for 30 minutes on ice. $3 \mathrm{~mL}$ FACS buffer was added to each tube after 30 minutes and tubes were centrifuged at $300 \mathrm{~g}$ for 3 minutes at $4^{\circ} \mathrm{C}$. Cells were washed again with $3 \mathrm{~mL}$ FACS buffer and centrifuged at $300 \mathrm{~g}$ for 3 minutes at $4^{\circ} \mathrm{C}$. Secondary antibodies were incubated for 30 minutes on ice. $3 \mathrm{~mL}$ FACS buffer was added to each tube after 30 minutes and tubes were centrifuged at $300 \mathrm{~g}$ for 3 minutes at $4^{\circ} \mathrm{C}$. Cells were washed again with $3 \mathrm{~mL}$ FACS buffer and centrifuged at $300 \mathrm{~g}$ for 3 minutes at $4^{\circ} \mathrm{C}$. Cells were resuspended in FACS buffer and $0.2 \mu \mathrm{g} / \mathrm{mL}$ DAPI was added to respective tubes. FACS was performed using a Sony MA900 cell sorter and accompanying software. Cells were collected in $1 \mathrm{~mL} 3 \mathrm{~F}$ media $+10 \mu \mathrm{M} Y-27632$. Secondary antibodies were purchased from Jackson Immuno and used at a dilution of 1:500.

\section{ACKNOWLEDGEMENTS}

We would like to thank the University of Michigan Advanced Genomics core for the operation of the 10X Chromium single cell capture platform, the University of Michigan Microscopy core for providing access to confocal microscopes and the Flow Cytometry core for providing access to flow cytometers.

\section{COMPETING INTERESTS}

JRS holds intellectual property pertaining to lung organoid technologies.

\section{FUNDING}

JRS is supported by the Cystic Fibrosis Foundation Epithelial Stem Cell Consortium, by grant CZF2019002440 from the Chan Zuckerberg Initiative DAF, an advised fund of the Silicon Valley Community Foundation, and by the National Heart, Lung, and Blood Institute (NHLBI; R01HL119215). RFCH was supported by a NIH Tissue Engineering and Regenerative Medicine Training Grant (NIH-NIDCR T32DE007057) and by a Ruth L. Kirschstein Predoctoral Individual National Research Service Award (NIH-NHLBI F31HL152531). ASC was supported by the T32 Michigan Medical Scientist Training Program (5T32GM007863-40) and by a Ruth L. Kirschstein Predoctoral Individual National Research Service Award (NIH-NHLBI F30HL156474). TF was supported by a NIH Tissue Engineering and Regenerative 
Medicine Training Grant (NIH-NIDCR T32DE007057). EMH was supported by a Ruth L. Kirschstein Predoctoral Individual National Research Service Award (NIH-NHBLI F31HL146162).

\section{AUTHOR CONTRIBUTIONS}

$\mathrm{RFCH}, \mathrm{ASC}$ and JRS conceived the study. JRS supervised the research. RFCH, ASC and AF designed, performed, and interpreted experiments optimizing and characterizing NKX2-1+ spheroids, LPOs and iBTOs and involving iAirO and iAlvO differentiations. ZX and TF performed computational analysis on scRNA-seq data, and RFCH, ASC and JRS interpreted scRNA-seq results. RFCH, ASC, Y-HT and EMH developed and executed tissue dissociation methods for FACS and SCRNA-seq and operated flow cytometers. RFCH, ASC and CJC performed whole mount staining and imaging. SH developed reagents and protocols for hPSC culture and differentiations. JM provided the NKX2-1-EGFP/TP63-mCherry iPSC reporter line and provide insight into the study. RFCH, ASC and JRS assembled figures and wrote the manuscript. ZX contributed to writing the methods. All authors read, contributed feedback, and approved the manuscript.

\section{DATA AVAILABILTY}

Sequencing data used in this study is deposited at EMBL-EBI ArrayExpress. Single-cell RNA sequencing of human fetal lung and human fetal bud tip organoids: human fetal lung (ArrayExpress: E-MTAB-8221, ArrayExpress: E-MTAB-10662) (Miller et al., 2020; Hein et al., 2021), spheroids, LPOs and iBTOs (in progress) (this study). Code used to process data can be found at: https://github.com/jason-spencelab/Hein_Conchola_2022.

\section{REFERENCES}

Abler, L. L. et al. (2017) 'Human embryonic lung epithelial tips are multipotent progenitors that can be expanded in vitro as long-term self-renewing organoids', Development. American Physiological Society, 4(1), p. e26575. doi: 10.7554/eLife.26575.

Abler, L. L., Mansour, S. L. and Sun, X. (2008) 'Conditional gene inactivation reveals roles for Fgf10 and Fgfr2 in establishing a normal pattern of epithelial branching in the mouse lung', Developmental Dynamics, 238(8), pp. 1999-2013. doi: 10.1002/dvdy.22032.

Alanis, D. M. et al. (2014) 'Two nested developmental waves demarcate a compartment boundary in the mouse lung', Nature Communications. Nature Publishing Group, 5(1), p. 3923. doi:

10.1038/ncomms4923.

Alysandratos, K.-D. et al. (2022) 'Impact of cell culture on the transcriptomic programs of primary and iPSC-derived human alveolar type 2 cells', bioRxiv. Cold Spring Harbor Laboratory, p.

2022.02.08.479591. doi: 10.1101/2022.02.08.479591.

Aros, C. J., Pantoja, C. J. and Gomperts, B. N. (2021) 'Wnt signaling in lung development, regeneration, and disease progression', Communications Biology 2021 4:1. Nature Publishing Group, 4(1), pp. 1-13. 
doi: 10.1038/s42003-021-02118-w.

Becht, E. et al. (2019) 'Dimensionality reduction for visualizing single-cell data using UMAP', Nature Biotechnology. Nature Publishing Group, 37(1), pp. 38-44. doi: 10.1038/nbt.4314.

Blondel, V. D. et al. (2008) 'Fast unfolding of communities in large networks', Journal of Statistical Mechanics: Theory and Experiment, 2008(10). doi: 10.1088/1742-5468/2008/10/P10008.

Bogue, C. W. et al. (1998) 'Hex Expression During Development Suggests an Important Role in Both Gastrulation and Organogenesis • 251', Pediatric Research 1998 43:4. Nature Publishing Group, 43(4), pp. 45-45. doi: 10.1203/00006450-199804001-00272.

Carraro, G. et al. (2021) 'Transcriptional analysis of cystic fibrosis airways at single-cell resolution reveals altered epithelial cell states and composition', Nature Medicine 2021 27:5. Nature Publishing Group, 27(5), pp. 806-814. doi: 10.1038/s41591-021-01332-7. de Carvalho, A. L. R. T. et al. (2019) 'Glycogen synthase kinase 3 induces multilineage maturation of human pluripotent stem cell-derived lung progenitors in 3D culture.', Development (Cambridge, England). Oxford University Press for The Company of Biologists Limited, 146(2), p. dev171652. doi: 10.1242/dev.171652.

Chen, Y.-W. et al. (2017) 'A three-dimensional model of human lung development and disease from pluripotent stem cells', Nature Cell Biology. Nature Publishing Group, 19(5), pp. 542-549. doi: 10.1038/ncb3510.

Chin, A. M. et al. (2016) 'A Dynamic WNT/B-CATENIN Signaling Environment Leads to WNTIndependent and WNT-Dependent Proliferation of Embryonic Intestinal Progenitor Cells', Stem Cell Reports. Cell Press, 7(5), pp. 826-839. doi: 10.1016/J.STEMCR.2016.09.004/ATTACHMENT/29E9B5805C50-4DF3-B3B1-3B16564B4215/MMC1.PDF.

Conway, R. F. et al. (2020) 'Understanding Human Lung Development through In Vitro Model Systems', BioEssays. John Wiley and Sons Inc., 42(6). doi: 10.1002/bies.202000006.

Crane, A. M. et al. (2015) 'Targeted correction and restored function of the CFTR gene in cystic fibrosis induced pluripotent stem cells', Stem Cell Reports. Cell Press, 4(4), pp. 569-577. doi:

10.1016/J.STEMCR.2015.02.005/ATTACHMENT/B791AC47-2A8A-4089-885A233B1EE7974B/MMC1.PDF.

D'Amour, K. A. et al. (2006) 'Production of pancreatic hormone-expressing endocrine cells from human embryonic stem cells', Nature Biotechnology. Nature Publishing Group, 24(11), pp. 1392-1401. doi: $10.1038 / \mathrm{nbt} 1259$.

Davenport, C. et al. (2016) 'Anterior-Posterior Patterning of Definitive Endoderm Generated from Human Embryonic Stem Cells Depends on the Differential Signaling of Retinoic Acid, Wnt-, and BMP-Signaling', STEM CELLS. John Wiley \& Sons, Ltd, 34(11), pp. 2635-2647. doi: 10.1002/stem.2428.

Domyan, E. T. et al. (2011) 'Signaling through BMP receptors promotes respiratory identity in the foregut via repression of Sox2.', Development (Cambridge, England). Company of Biologists, 138(5), pp. 971-81. doi: 10.1242/dev.053694. 
Dye, B. R. et al. (2015) 'In vitro generation of human pluripotent stem cell derived lung organoids', eLife. eLife Sciences Publications Limited, 4, p. e05098. doi: 10.7554/eLife.05098.

Dye, B. R. et al. (2016) 'A bioengineered niche promotes in vivo engraftment and maturation of pluripotent stem cell derived human lung organoids', eLife. eLife Sciences Publications Limited, 5, p. e19732. doi: 10.7554/eLife.19732.

Gadue, P. et al. (2006) 'Wnt and TGF-beta signaling are required for the induction of an in vitro model of primitive streak formation using embryonic stem cells.', Proceedings of the National Academy of Sciences of the United States of America. National Academy of Sciences, 103(45), pp. 16806-11. doi:

10.1073/pnas.0603916103.

Goss, A. M. et al. (2009) 'Wnt2/2b and $\beta$-catenin signaling are necessary and sufficient to specify lung progenitors in the foregut', Developmental cell. NIH Public Access, 17(2), p. 290. doi:

10.1016/J.DEVCEL.2009.06.005.

Gotoh, S. et al. (2014) 'Generation of alveolar epithelial spheroids via isolated progenitor cells from human pluripotent stem cells.', Stem cell reports. Elsevier, 3(3), pp. 394-403. doi:

10.1016/j.stemcr.2014.07.005.

Green, M. D. et al. (2011) 'Generation of anterior foregut endoderm from human embryonic and induced pluripotent stem cells.', Nature biotechnology. NIH Public Access, 29(3), pp. 267-72. doi:

10.1038/nbt. 1788 .

Hafemeister, C. and Satija, R. (2019) 'Normalization and variance stabilization of single-cell RNA-seq data using regularized negative binomial regression', Genome Biology. BioMed Central Ltd., 20(1), pp. 115. doi: 10.1186/S13059-019-1874-1/FIGURES/6.

Han, L. et al. (2020) 'Single cell transcriptomics identifies a signaling network coordinating endoderm and mesoderm diversification during foregut organogenesis', Nature Communications 2020 11:1. Nature Publishing Group, 11(1), pp. 1-16. doi: 10.1038/s41467-020-17968-x.

Hao, Y. et al. (2021) 'Integrated analysis of multimodal single-cell data', Cell. Cell, 184(13), pp. 35733587.e29. doi: 10.1016/J.CELL.2021.04.048.

Harris-Johnson, K. S. et al. (2009) 'beta-Catenin promotes respiratory progenitor identity in mouse foregut.', Proceedings of the National Academy of Sciences of the United States of America. National Academy of Sciences, 106(38), pp. 16287-92. doi: 10.1073/pnas.0902274106.

Hawkins, F. et al. (2017) 'Prospective isolation of NKX2-1-expressing human lung progenitors derived from pluripotent stem cells', The Journal of Clinical Investigation. American Society for Clinical Investigation, 127(6), pp. 2277-2294. doi: 10.1172/JCl89950.

Hawkins, F. J. et al. (2021) 'Derivation of Airway Basal Stem Cells from Human Pluripotent Stem Cells', Cell stem cell. NIH Public Access, 28(1), p. 79. doi: 10.1016/J.STEM.2020.09.017.

Heemskerk, I. et al. (2019) 'Rapid changes in morphogen concentration control self-organized patterning in human embryonic stem cells', eLife. eLife Sciences Publications Ltd, 8. doi: 10.7554/ELIFE.40526. Hein, R. F. C. et al. (2021) 'R-SPONDIN2+ Mesenchymal Cells Form the Bud Tip Progenitor Niche 
During Human Lung Development', bioRxiv. Cold Spring Harbor Laboratory, p. 2021.04.05.438484. doi: 10.1101/2021.04.05.438484.

Holloway, E. M. et al. (2020) 'Differentiation of Human Intestinal Organoids with Endogenous Vascular Endothelial Cells', Developmental Cell. Cell Press, 54(4), pp. 516-528.e7. doi:

10.1016/j.devcel.2020.07.023.

Hor, P. et al. (2020) 'Efficient Generation and Transcriptomic Profiling of Human iPSC-Derived Pulmonary Neuroendocrine Cells', iScience. iScience, 23(5). doi: 10.1016/J.ISCI.2020.101083.

Huang, S. X. L. et al. (2014) 'Efficient generation of lung and airway epithelial cells from human pluripotent stem cells', Nature Biotechnology. Nature Publishing Group, 32(1), pp. 84-91. doi: $10.1038 / n b t .2754$.

Hurley, K. et al. (2020) 'Reconstructed Single-Cell Fate Trajectories Define Lineage Plasticity Windows during Differentiation of Human PSC-Derived Distal Lung Progenitors', Cell Stem Cell. Cell Press. doi: 10.1016/J.STEM.2019.12.009.

Jacob, A. et al. (2017) 'Differentiation of Human Pluripotent Stem Cells into Functional Lung Alveolar Epithelial Cells', Cell Stem Cell. Cell Press, 21(4), pp. 472-488.e10. doi: 10.1016/J.STEM.2017.08.014. Jacob, A. et al. (2019) 'Derivation of self-renewing lung alveolar epithelial type II cells from human pluripotent stem cells', Nature Protocols 2019 14:12. Nature Publishing Group, 14(12), pp. 3303-3332. doi: 10.1038/s41596-019-0220-0.

Jacobs, I. J., Ku, W.-Y. and Que, J. (2012) 'Genetic and cellular mechanisms regulating anterior foregut and esophageal development', Developmental Biology. Academic Press, 369(1), pp. 54-64. doi:

10.1016/J.YDBIO.2012.06.016.

Jo, K. et al. (2021) 'Efficient differentiation of human primordial germ cells through geometric control reveals a key role for NODAL signaling', bioRxiv. Cold Spring Harbor Laboratory, p. 2021.08.04.455129. doi: 10.1101/2021.08.04.455129.

Kapoor, A., Li, H. J. and Leiter, A. B. (2007) 'Intestinal Development: The Many Faces of Wnt Signaling', Gastroenterology. Elsevier, 133(2), pp. 710-712. doi: 10.1053/J.GASTRO.2007.06.029.

Kearns, N. A. et al. (2013) 'Generation of organized anterior foregut epithelia from pluripotent stem cells using small molecules', Stem Cell Research. Elsevier, 11(3), pp. 1003-1012. doi:

10.1016/J.SCR.2013.06.007.

Konishi, S. et al. (2016) 'Directed Induction of Functional Multi-ciliated Cells in Proximal Airway Epithelial Spheroids from Human Pluripotent Stem Cells', Stem Cell Reports. The Authors, 6(1), pp. 18-25. doi: 10.1016/j.stemcr.2015.11.010.

Kreitzer, F. R. et al. (2013) 'A robust method to derive functional neural crest cells from human pluripotent stem cells', American Journal of Stem Cells. e-Century Publishing Corporation, 2(2), p. 119. Available at: /pmc/articles/PMC3708511/ (Accessed: 15 February 2022).

Kubo, A. et al. (2004) 'Development of definitive endoderm from embryonic stem cells in culture.', Development (Cambridge, England). Oxford University Press for The Company of Biologists Limited, 
131(7), pp. 1651-62. doi: 10.1242/dev.01044.

Kuwahara, A. et al. (2020) 'Delineating the early transcriptional specification of the mammalian trachea and esophagus', eLife. eLife Sciences Publications Ltd, 9, pp. 1-23. doi: 10.7554/eLife.55526.

Lazzaro, D. et al. (1991) 'The transcription factor TTF-1 is expressed at the onset of thyroid and lung morphogenesis and in restricted regions of the foetal brain.', Development (Cambridge, England), 113(4), pp. 1093-104. Available at: http://www.ncbi.nlm.nih.gov/pubmed/1811929 (Accessed: 10 September 2019).

Leibel, S. L. et al. (2020) 'Generation of Complete Multi-Cell Type Lung Organoids From Human Embryonic and Patient-Specific Induced Pluripotent Stem Cells for Infectious Disease Modeling and Therapeutics Validation.', Current protocols in stem cell biology. Curr Protoc Stem Cell Biol, 54(1), p. e118. doi: 10.1002/cpsc.118.

$\mathrm{Li}, \mathrm{S}$. et al. (2016) 'Foxp transcription factors suppress a non-pulmonary gene expression program to permit proper lung development', Developmental biology. NIH Public Access, 416(2), p. 338. doi: 10.1016/J.YDBIO.2016.06.020.

Little, D. R. et al. (2019) 'Transcriptional control of lung alveolar type 1 cell development and maintenance by NK homeobox 2-1', Proceedings of the National Academy of Sciences, 116(41), pp. 20545-20555. doi: 10.1073/pnas.1906663116.

Loh, K. M. et al. (2014) 'Efficient endoderm induction from human pluripotent stem cells by logically directing signals controlling lineage bifurcations.', Cell stem cell. NIH Public Access, 14(2), pp. 237-52. doi: 10.1016/j.stem.2013.12.007.

Martyn, I. et al. (2018) 'Self-organization of a human organizer by combined Wnt and Nodal signalling', Nature. Nature Publishing Group, 558(7708), pp. 132-135. doi: 10.1038/s41586-018-0150-y.

Matsuno, K. et al. (2016) 'Redefining definitive endoderm subtypes by robust induction of human induced pluripotent stem cells', Differentiation. Elsevier, 92(5), pp. 281-290. doi: 10.1016/J.DIFF.2016.04.002. McCauley, K. B. et al. (2017) 'Efficient Derivation of Functional Human Airway Epithelium from Pluripotent Stem Cells via Temporal Regulation of Wnt Signaling', Cell stem cell. Cell Stem Cell, 20(6), pp. 844857.e6. doi: 10.1016/J.STEM.2017.03.001.

McCauley, K. B. et al. (2018) 'Single-Cell Transcriptomic Profiling of Pluripotent Stem Cell-Derived SCGB3A2+ Airway Epithelium', Stem Cell Reports, 10(5), pp. 1579-1595. doi:

10.1016/j.stemcr.2018.03.013.

McCracken, K. W. et al. (2011) 'Generating human intestinal tissue from pluripotent stem cells in vitro', Nature Protocols. Nature Publishing Group, 6(12), pp. 1920-1928. doi: 10.1038/nprot.2011.410. McCracken, K. W. et al. (2014) 'Modeling human development and disease in pluripotent stem cellderived gastric organoids', Nature. NIH Public Access, 516(7531), p. 400. doi: 10.1038/NATURE13863. Mclnnes, L., Healy, J. and Melville, J. (2018) 'UMAP: Uniform Manifold Approximation and Projection for Dimension Reduction', arXiv. arXiv. Available at: http://arxiv.org/abs/1802.03426 (Accessed: 13 January 2021). 
Miller, A. J. et al. (2018) 'In Vitro Induction and In Vivo Engraftment of Lung Bud Tip Progenitor Cells Derived from Human Pluripotent Stem Cells.', Stem cell reports. Elsevier, 10(1), pp. 101-119. doi: 10.1016/j.stemcr.2017.11.012.

Miller, A. J. et al. (2019) 'Generation of lung organoids from human pluripotent stem cells in vitro', Nature Protocols. Nature Publishing Group, 14(2), pp. 518-540. doi: 10.1038/s41596-018-0104-8.

Miller, A. J. et al. (2020) 'In Vitro and In Vivo Development of the Human Airway at Single-Cell Resolution', Developmental Cell. Elsevier, 0(0). doi: 10.1016/j.devcel.2020.01.033.

Miller, M. F. et al. (2012) 'Wnt ligands signal in a cooperative manner to promote foregut organogenesis', Proceedings of the National Academy of Sciences of the United States of America, 109(38), pp. 1534815353. doi: 10.1073/pnas.1201583109.

Minoo, P. et al. (1995) 'TTF-1 Regulates Lung Epithelial Morphogenesis', Developmental Biology, 172(2), pp. 694-698. doi: 10.1006/dbio.1995.8080.

Minoo, P. et al. (1999) 'Defects in Tracheoesophageal and Lung Morphogenesis inNkx2.1(-/-) Mouse Embryos', Developmental Biology. Academic Press, 209(1), pp. 60-71. doi: 10.1006/DBIO.1999.9234. Mou, H. et al. (2012) 'Generation of multipotent lung and airway progenitors from mouse ESCs and patient-specific cystic fibrosis iPSCs.', Cell stem cell. NIH Public Access, 10(4), pp. 385-97. doi: 10.1016/j.stem.2012.01.018.

Nikolić, M. Z. et al. (2017) 'Human embryonic lung epithelial tips are multipotent progenitors that can be expanded in vitro as long-term self-renewing organoids.', eLife. eLife Sciences Publications, Ltd, 6. doi: 10.7554/eLife.26575.

Nikolić, M. Z. and Rawlins, E. L. (2017) 'Lung Organoids and Their Use To Study Cell-Cell Interaction', Current Pathobiology Reports. Springer US, 5(2), pp. 223-231. doi: 10.1007/s40139-017-0137-7.

Okubo, T. and Hogan, B. L. M. (2004) 'Hyperactive Wnt signaling changes the developmental potential of embryonic lung endoderm', Journal of biology. J Biol, 3(3). doi: 10.1186/JBIOL3.

Perl, A. K. T. et al. (2005) 'Normal lung development and function after Sox9 inactivation in the respiratory epithelium', Genesis (New York, N.Y. : 2000). Genesis, 41(1), pp. 23-32. doi: 10.1002/GENE.20093. Pinto, D. and Clevers, H. (2005) 'Wnt control of stem cells and differentiation in the intestinal epithelium', Experimental cell research. Exp Cell Res, 306(2), pp. 357-363. doi: 10.1016/J.YEXCR.2005.02.022. Que, J. et al. (2007) 'Multiple dose-dependent roles for Sox2 in the patterning and differentiation of anterior foregut endoderm.', Development (Cambridge, England). The Company of Biologists Ltd, 134(13), pp. 2521-31. doi: 10.1242/dev.003855.

Rabata, A. et al. (2020) '3D cell culture models demonstrate a role for FGF and WNT signaling in regulation of lung epithelial cell fate and morphogenesis', bioRxiv. Cold Spring Harbor Laboratory, $p$. 2020.04.04.024943. doi: 10.1101/2020.04.04.024943.

Rankin, S. A. et al. (2016) 'A Retinoic Acid-Hedgehog Cascade Coordinates Mesoderm-Inducing Signals and Endoderm Competence during Lung Specification.', Cell reports. Elsevier, 16(1), pp. 66-78. doi: 10.1016/j.celrep.2016.05.060. 
Rankin, S. A. et al. (2018) 'Timing is everything: Reiterative Wnt, BMP and RA signaling regulate developmental competence during endoderm organogenesis', Developmental Biology. Academic Press, 434(1), pp. 121-132. doi: 10.1016/J.YDBIO.2017.11.018.

Rawlins, E. L. et al. (2009) 'The Id2+ distal tip lung epithelium contains individual multipotent embryonic progenitor cells', Development, 136(22), pp. 3741-3745. doi: 10.1242/dev.037317.

Rockich, B. E. et al. (2013) 'Sox9 plays multiple roles in the lung epithelium during branching morphogenesis', Proceedings of the National Academy of Sciences, 110(47), pp. E4456-E4464. doi: 10.1073/pnas.1311847110.

Serra, M. et al. (2017) 'Pluripotent stem cell differentiation reveals distinct developmental pathways regulating lung- versus thyroid-lineage specification.', Development (Cambridge, England). Oxford University Press for The Company of Biologists Limited, 144(21), pp. 3879-3893. doi: 10.1242/dev.150193.

Serra, R., Pelton, R. W. and Moses, H. L. (1994) 'TGF beta 1 inhibits branching morphogenesis and Nmyc expression in lung bud organ cultures', Development. The Company of Biologists, 120(8), pp. 21532161. doi: 10.1242/DEV.120.8.2153.

Shu, W. et al. (2005) 'Wnt/ß-catenin signaling acts upstream of N-myc, BMP4, and FGF signaling to regulate proximal-distal patterning in the lung', Developmental Biology. Academic Press, 283(1), pp. 226-239. doi: 10.1016/J.YDBIO.2005.04.014.

Shu, W. et al. (2007) 'Foxp2 and Foxp1 cooperatively regulate lung and esophagus development', Development. The Company of Biologists, 134(10), pp. 1991-2000. doi: 10.1242/DEV.02846. Spence, J. R. et al. (2009) 'Sox17 Regulates Organ Lineage Segregation of Ventral Foregut Progenitor Cells', Developmental Cell. Cell Press, 17(1), pp. 62-74. doi: 10.1016/J.DEVCEL.2009.05.012. Spence, J. R. et al. (2011) 'Directed differentiation of human pluripotent stem cells into intestinal tissue in vitro.', Nature. NIH Public Access, 470(7332), pp. 105-9. doi: 10.1038/nature09691.

Stuart, T. et al. (2019) 'Comprehensive Integration of Single-Cell Data', Cell. Cell Press, 177(7), pp. 18881902.e21. doi: 10.1016/J.CELL.2019.05.031/ATTACHMENT/2F8B9EBE-54E6-43EB-9EF2949B6BDA8BA2/MMC3.PDF.

Sun, D., Evans, L. D. and Rawlins, E. L. (2020) 'Organoid Easytag: an efficient workflow for gene targeting in human organoids', bioRxiv. Cold Spring Harbor Laboratory, p. 2020.05.04.076067. doi: 10.1101/2020.05.04.076067.

Tamò, L. et al. (2018) 'Generation of an alveolar epithelial type II cell line from induced pluripotent stem cells', American Journal of Physiology-Lung Cellular and Molecular Physiology, 315(6), pp. L921-L932. doi: 10.1152/ajplung.00357.2017.

Traag, V. A., Waltman, L. and van Eck, N. J. (2019) 'From Louvain to Leiden: guaranteeing wellconnected communities', Scientific Reports 2019 9:1. Nature Publishing Group, 9(1), pp. 1-12. doi: 10.1038/s41598-019-41695-z.

Volckaert, T. and De Langhe, S. P. (2015) 'Wnt and FGF mediated epithelial-mesenchymal crosstalk 
during lung development', Developmental Dynamics. Wiley-Blackwell, 244(3), pp. 342-366. doi: $10.1002 / d v d y .24234$.

Wang, D. et al. (2007) 'A pure population of lung alveolar epithelial type II cells derived from human embryonic stem cells.', Proceedings of the National Academy of Sciences of the United States of America. National Academy of Sciences, 104(11), pp. 4449-54. doi: 10.1073/pnas.0700052104. Yamamoto, Y. et al. (2017) 'Long-term expansion of alveolar stem cells derived from human iPS cells in organoids.', Nature methods, 14(11), pp. 1097-1106. doi: 10.1038/nmeth.4448.

Yamamoto, Y. et al. (2020) 'A method of generating alveolar organoids using human pluripotent stem cells', Methods in Cell Biology. Academic Press, 159, pp. 115-141. doi: 10.1016/BS.MCB.2020.02.004. Yan, K. S. et al. (2017) 'Non-equivalence of Wnt and R-spondin ligands during Lgr5+ intestinal stem-cell self-renewal.', Nature. NIH Public Access, 545(7653), pp. 238-242. doi: 10.1038/nature22313.

Yu, Q. et al. (2020) 'An organoid and multi-organ developmental cell atlas reveals multilineage fate specification in the human intestine', bioRxiv. Cold Spring Harbor Laboratory, p. 2020.07.24.219147. doi: 10.1101/2020.07.24.219147. 
bioRxiv preprint doi: https://doi.org/10.1101/2022.02.25.481981; this version posted February 25, 2022. The copyright holder for this preprint (which was not certified by peer review) is the author/funder, who has granted bioRxiv a license to display the preprint in perpetuity. It is made available under aCC-BY-NC-ND 4.0 International license.

\section{Figure 1}

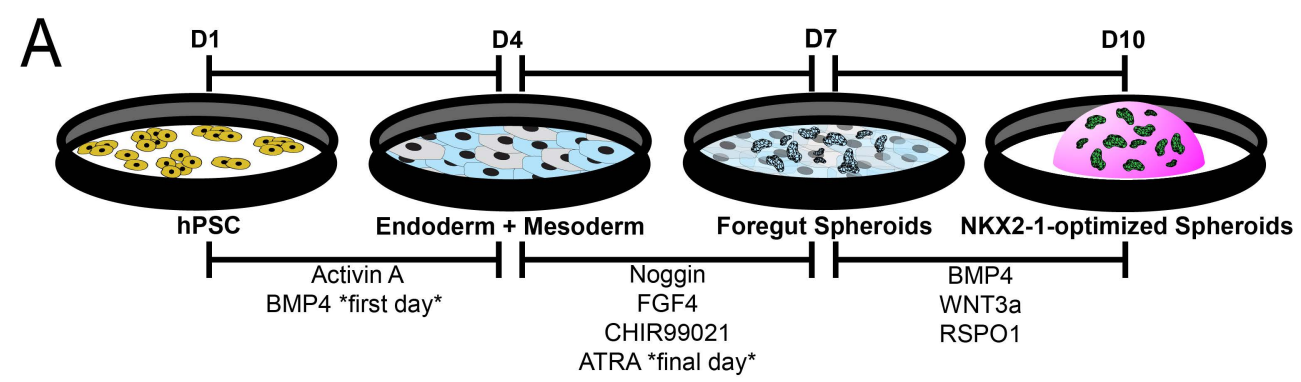

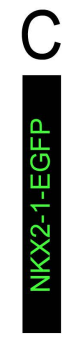
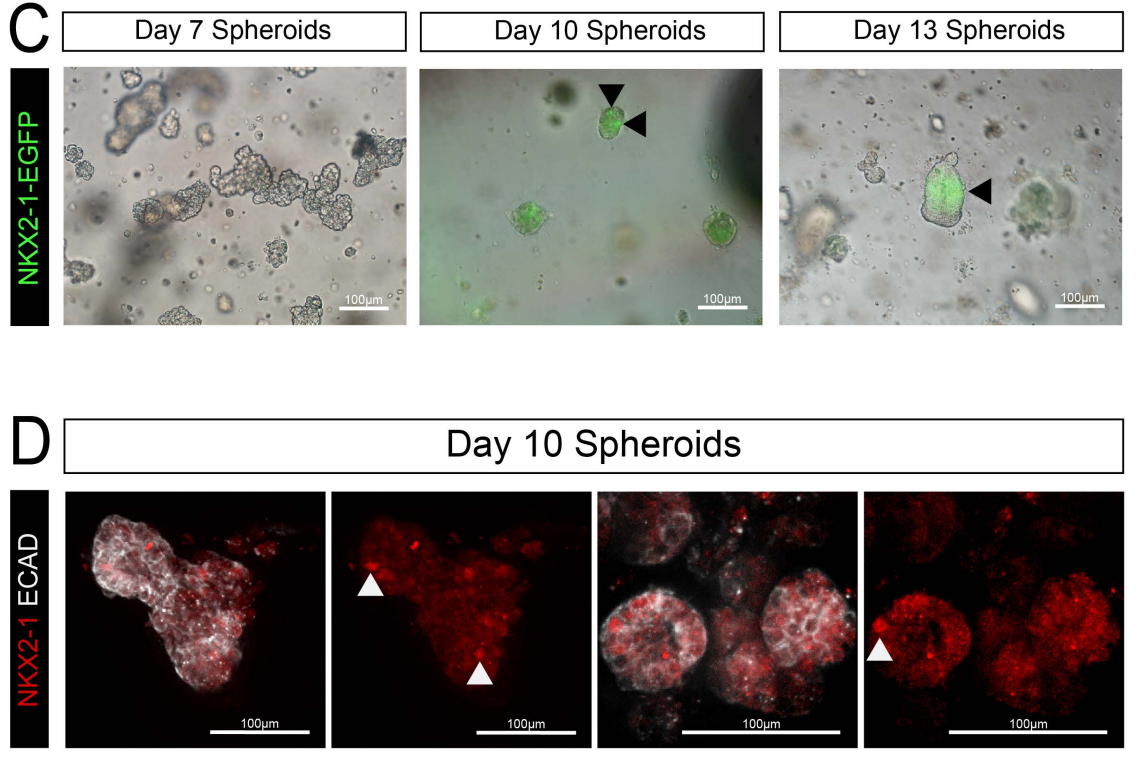

$\mathrm{E}$
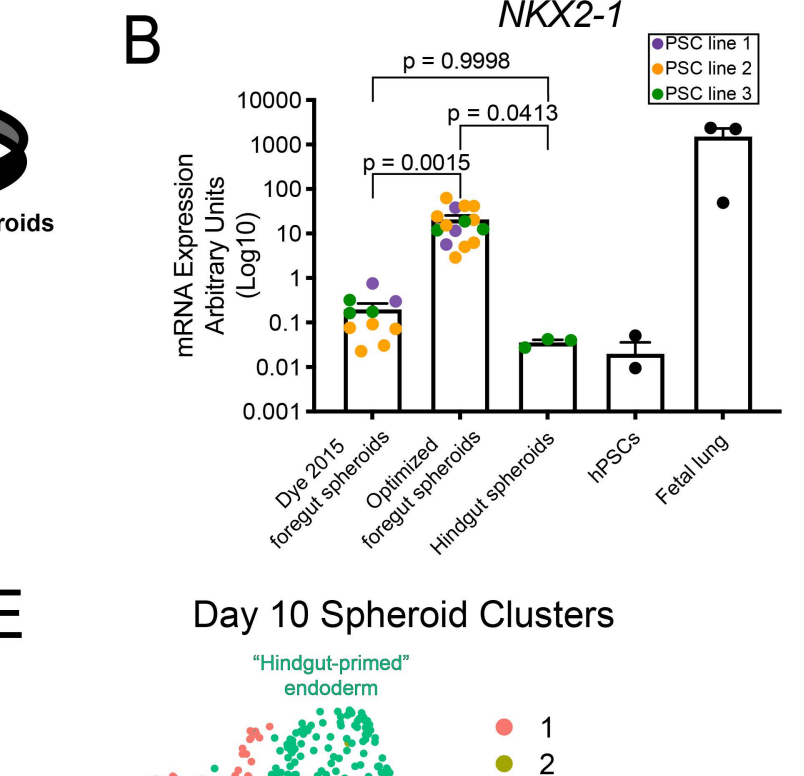

F

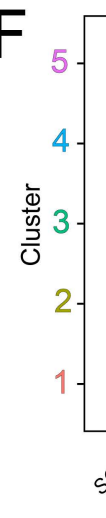

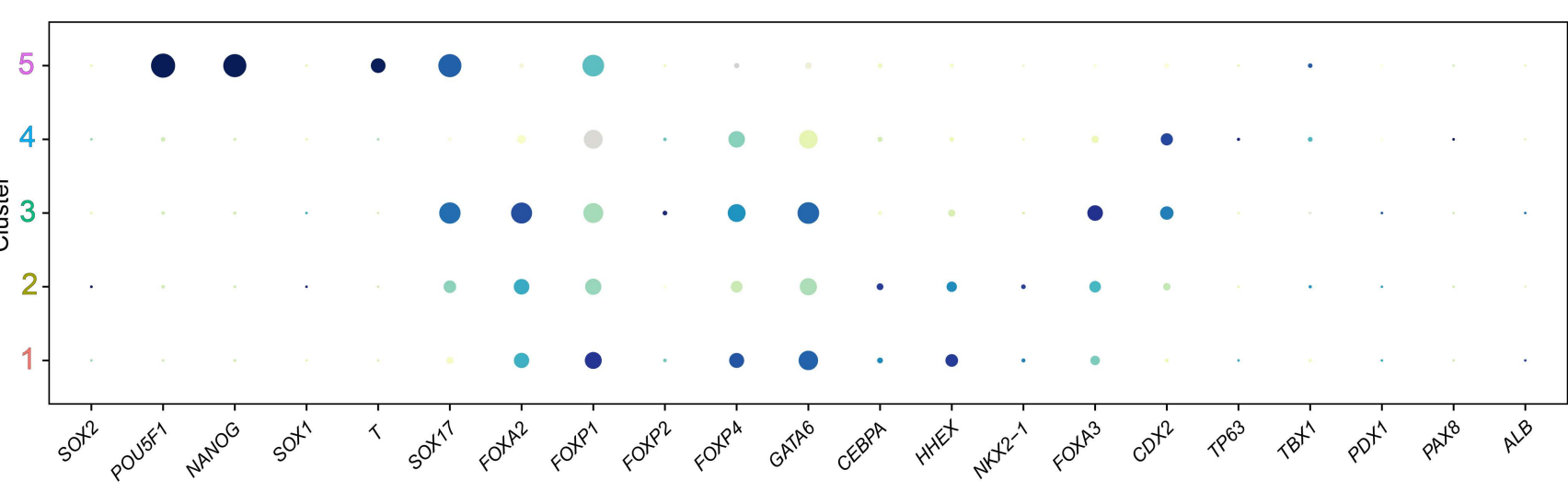

Percent Expressed

. 0

$\circ 25$

050

O75

Average Expression

Highest 


\section{Figure 1: Optimization of Lung Spheroids for NKX2-1 Expression}

(A) Schematic displaying the directed differentiation protocol from hPSC to NKX2-1-optimized spheroids.

(B) RT-qPCR data comparing NKX2-1 expression in previously published foregut spheroids (Dye et al., 2015) to optimized foregut spheroids and hindgut spheroids (Spence et al., 2011). iPSCs and whole fetal lung are also included as references. Each color represents an independent experiment with a unique iPSC line (purple: WTC11, orange: iPSC17 WT 7B2, green: iPSC line 72.3). Each data point of the same color represents a technical replicate from the same iPSC line in one or more independent experiments. Error bars represent standard error of the mean. Statistical tests were performed by ordinary one-way ANOVA followed by Turkey's multiple comparison test.

(C) Representative reporter expression for NXK2-1-EGFP on day 7, 10, or 14 spheroids.

(D) Maximum intensity projection of a whole mount immunofluorescence confocal z-series staining for the pan-epithelial marker ECAD and lung epithelial marker NKX2-1.

(E) UMAP cluster plot of scRNA-seq data from day 10 spheroids $(n=1)$. Each dot represents a single cell and cells were computationally clustered based on transcriptional similarities. The plot is colored and numbered by cluster. Cell-type labels for each cluster are based on expression of canonical cell-type markers displayed in the dot plot in Fig. 1E or Fig. S1G.

(F) Dot plot of cell lineage genes in each cluster of the UMAP plot in Fig. 1D. The dot size represents the percentage of cells expressing the gene in the corresponding cluster, and the dot color indicates log-normalized expression level of the gene. 
bioRxiv preprint doi: https://doi.org/10.1101/2022.02.25.481981; this version posted February 25, 2022. The copyright holder for this preprint (which was not certified by peer review) is the author/funder, who has granted bioRxiv a license to display the preprint in perpetuity. It is made Figure 2

A

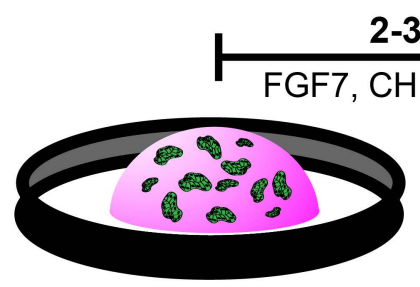

NKX2-1+ Lung Spheroids available under aCC-BY-NC-ND 4.0 International license. 


\section{Figure 2: iPSC-derived Bud Tip Progenitors Emerge Over Time in LPOs}

(A) Schematic displaying the Lung Progenitor Organoid (LPO) expansion protocol from NKX2-1optimized spheroids. LPOs form after $2-3$ weeks in culture.

(B) Brightfield image of 6-week LPO on inverted microscope.

(C) Representative NKX2-1-EGFP reporter images of 10 - 11-week LPOs, passaged whole or sheared (pipette and needle).

(D) Immunofluorescence staining on paraffin sections of the lung-like regions of 12-week LPOs for bud tip progenitor markers CPM and SOX9 and either lung epithelial markers NKX2-1 (left panels) or SOX2 (right panels).

(E) Immunofluorescence staining on paraffin sections for intestinal epithelial marker CDX2, intestinal goblet cell marker MUC2, and lung epithelial marker NKX2-1 on non-lung regions of 12-week LPOs.

(F) FACS quantification of NKX2-1-EGFP ${ }^{+} / \mathrm{CPM}^{+}$cells in LPOs in aggregate time course from the NKX2-1-EGFP reporter cell line. LPOs were sorted using the NKX2-1-EGFP reporter and bud tip progenitor cell surface marker CPM from $3-17$ weeks in 3F media. Percentages of live cells expressing neither marker (Negative, grey), each separate marker ( $\mathrm{CPM}^{+}$-only, blue; EGFP+-only, green), or dual-expressing cells $\left(\mathrm{CPM}^{+} / \mathrm{EGFP}^{+}\right.$, purple) are reported as mean $\pm \mathrm{SEM}$ for $2-3$ replicates per time point.

(G) UMAP cluster plot of scRNA-seq data from LPOs ( $n=2$ biological replicates for 3- and 6-week timepoints, $n=1$ for 10-week timepoint). Each dot represents a single cell and cells were computationally clustered based on transcriptional similarities. The plot is colored and numbered by cluster. Cell-type labels for each cluster are based on expression of canonical cell-type markers displayed in the heat map in Fig. S3 and the dot plot and feature plots in Fig. $2 \mathrm{G}$ and Fig. $\mathrm{SE}$. Feature plots corresponding to the LPO cluster plot and displaying canonical bud tip progenitor markers (SOX9, CPM, ETV5, TESC) (Miller et al., 2018, 2020; Yamamoto et al., 2020), lung epithelial markers (NKX2-1, FOXA2, SOX2) and intestine epithelial marker (CDX2). The color of each dot in the feature plot indicates log-normalized expression level of the set of bud tip progenitor genes in the represented cell. 
bioRxiv preprint doi: https://doi.org/10.1101/2022.02.25.481981; this version posted February 25, 2022. The copyright holder for this preprint (which was not certified by peer review) is the author/funder, who has granted bioRxiv a license to display the preprint in perpetuity. It is made Figure 3

A

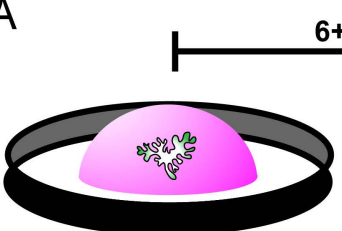

Lung Progenitor Organoid available under aCC-BY-NC-ND 4.0 International license. 
Figure 3: iPSC-derived Bud Tip Progenitors can be Isolated, Expanded Long-term and are Transcriptionally Similar to Human Fetal Bud Tip Progenitor Cultures

(A) Schematic displaying isolation and expansion of induced bud tip progenitor organoids (iBTOs). LPOs are maintained in 3F media and whole passaged for at least 6 weeks then are dissociated for FACS. iBTPs are isolated using $\mathrm{CPM}^{+}$expression +/- NKX2-1EGFP+ reporter expression and replated as isolated iBTPs. iBTPs re-form to iBTOs over 2-4 weeks, are maintained in 3F media, and whole passaged.

(B) Representative NKX2-1-EGFP reporter image of 3-week iBTO.

(C) Immunofluorescence staining on paraffin sections of nearly homogenous 4-week iBTOs for bud tip progenitor markers CPM and SOX9 and either lung epithelial markers NKX2-1 (left panels) or SOX2 (right panels).

(D) FACS quantification of NKX2-1-EGFP ${ }^{+} / \mathrm{CPM}^{+}$cells in iBTOs from 3-week sorted LPOs or 8 - 17week sorted LPOs from the NKX2-1-EGFP reporter cell line. iBTOs were analyzed $7-8$ weeks after iBTP purification from LPOs. Percentages of live cells expressing neither marker (Negative, grey), each separate marker ( $\mathrm{CPM}^{+}$-only, blue; EGFP+-only, green), or dual-expressing cells $\left(\mathrm{CPM}^{+} / \mathrm{EGFP}^{+}\right.$, purple $)$are reported as mean \pm SEM for $1-2$ replicates per time point.

(E) UMAP cluster plot of scRNA-seq data from iBTOs ( $n=1$ biological replicates for iBTOs from 4and 10-week LPOs). Each dot represents a single cell and cells were computationally clustered based on transcriptional similarities. The plot is colored and numbered by cluster. Cell-type labels for each cluster are based on expression of canonical cell-type markers displayed in the dot plots and feature plots in Fig. 3H and Fig. S4B.

(F) UMAP plot corresponding to the iBTO cluster plot in Fig. 3E. Each dot represents a single cell and dots/cells are colored by the sample from which they came from.

(G) Stacked bar graph displaying the proportion of cells from each sample in each cluster of the iBTO cluster plot in Fig. 3E.

(H) Feature plots and dot plot corresponding to the iBTO cluster plot in Fig. 3E and displaying canonical bud tip progenitor markers (SOX9, CPM, ETV5, TESC, FGF20, SOX11, HGMB2, NPC2, LGR5, ETV4) (Miller et al., 2018, 2020; Yamamoto et al., 2020), lung epithelial markers (NKX2-1, FOXA2, SOX2) and intestine epithelial marker (CDX2). The color of each dot in the feature plot indicates log-normalized expression level of the set of bud tip progenitor genes in the represented cell. For the dot plot, the dot size represents the percentage of cells expressing the gene in the corresponding cluster, and the dot color indicates log-normalized expression level of the gene.

(I) Violin plot displaying an in vivo bud tip progenitor cell score, calculated as the average expression of the top 50 enriched genes in in vivo bud tip progenitor cells, for each sample. Samples include whole LPOs (2x3-week LPOs, 2x6-week LPOs, 1x10-week LPOs), NXK2-1-extracted cells from 3-, 6-, and 10-week LPOs ( $\mathrm{n}=2$ for 3- and 6-week LPOs, $\mathrm{n}=1$ for 10-week LPOs), whole iBTOs 
(derived from LPOs sorted for NKX2-1 ${ }^{+} / \mathrm{CPM}^{+}$cells at 4 or 10 weeks, $\mathrm{n}=1$ of each), human fetalderived bud tip organoids (14-weeks post-conception) (Miller et al., 2020), and primary in vivo tissue (8.5 - 19-weeks post-conception), including computationally-extracted bud tip, basal, and neuroendocrine cells (Miller et al., 2020; Hein et al., 2021). The cell scoring list was generated from scRNA-seq data from the in vivo bud tip progenitor shown on the plot. 
Figure 4

A

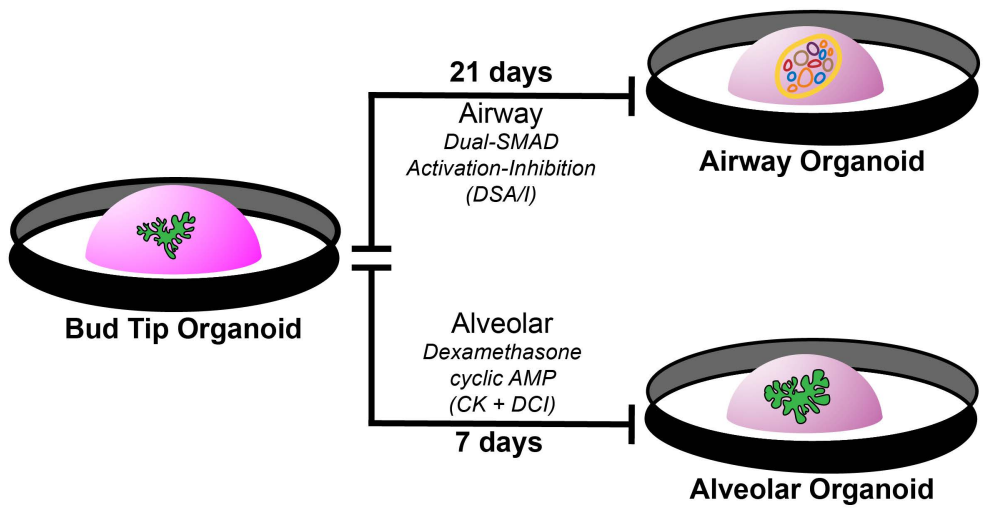

$\mathrm{C}$

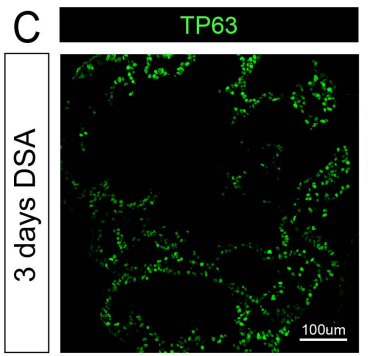

क

$\mathrm{D}$
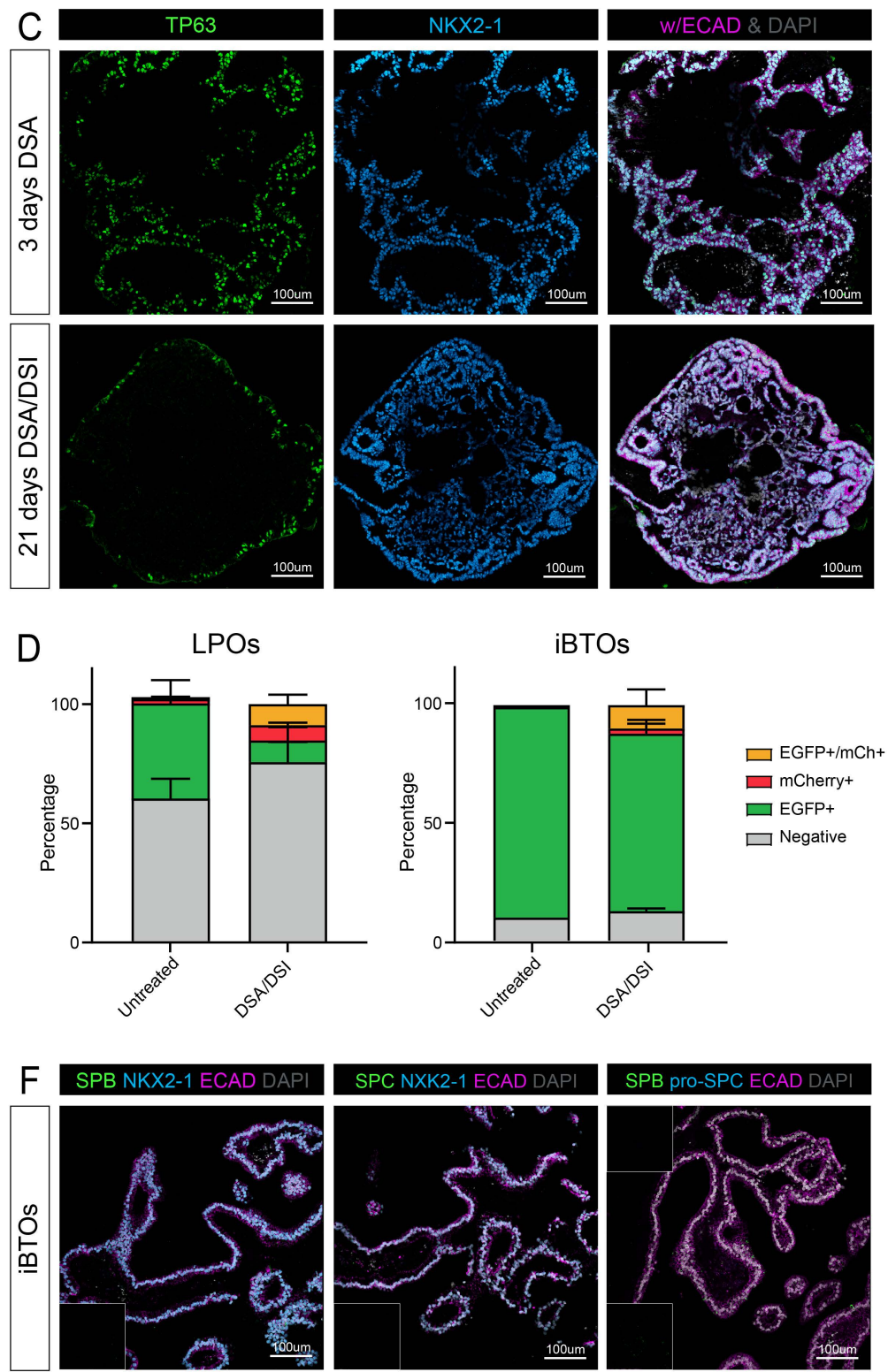

\begin{tabular}{l}
\hline \\
0 \\
0 \\
+ \\
$\frac{y}{0}$ \\
0 \\
$\frac{\pi}{0}$ \\
1 \\
1
\end{tabular}

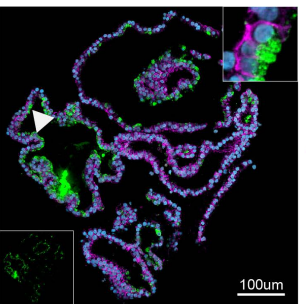

$\square$ EGFP+

$\square$ Negative

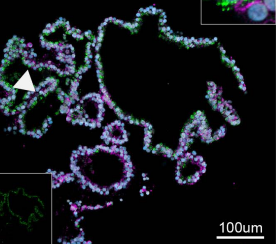

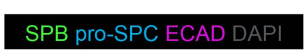
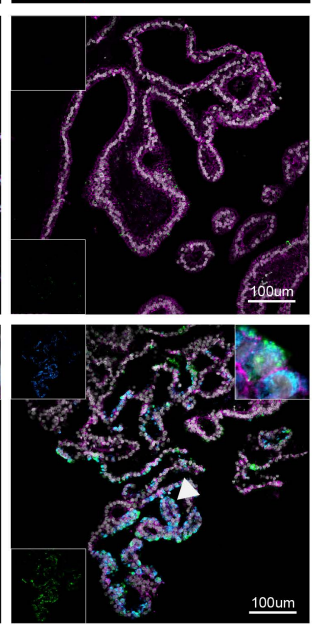
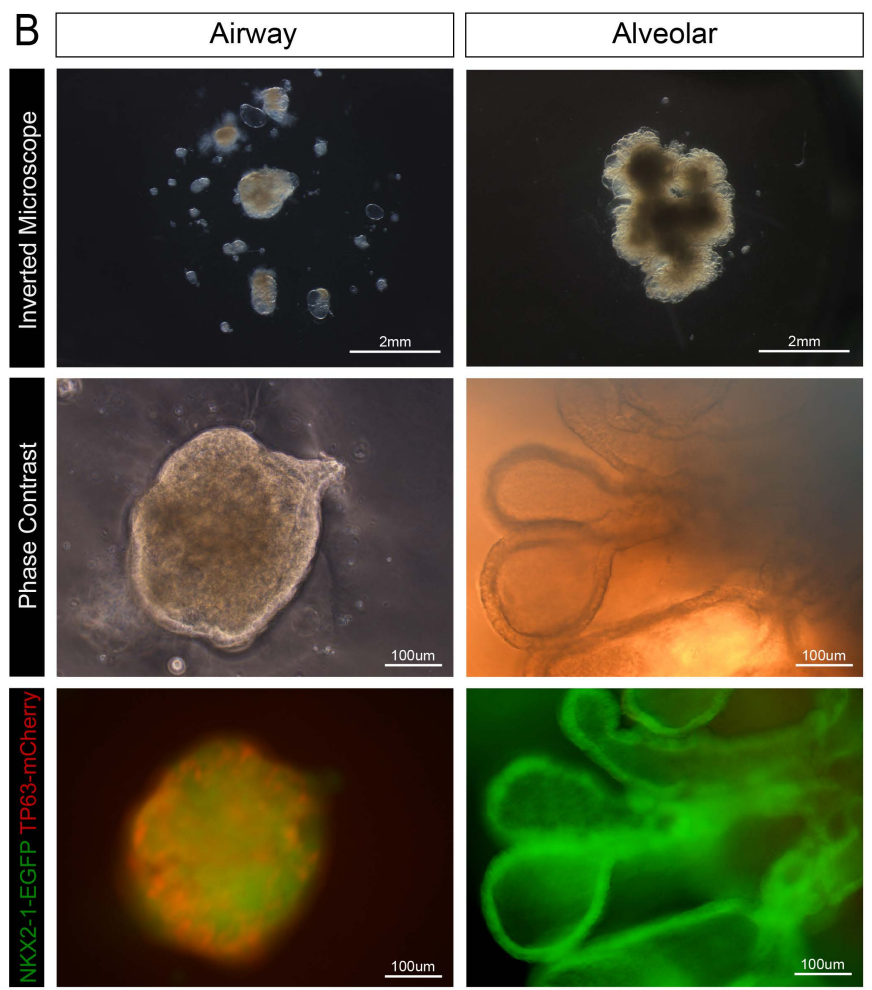

E
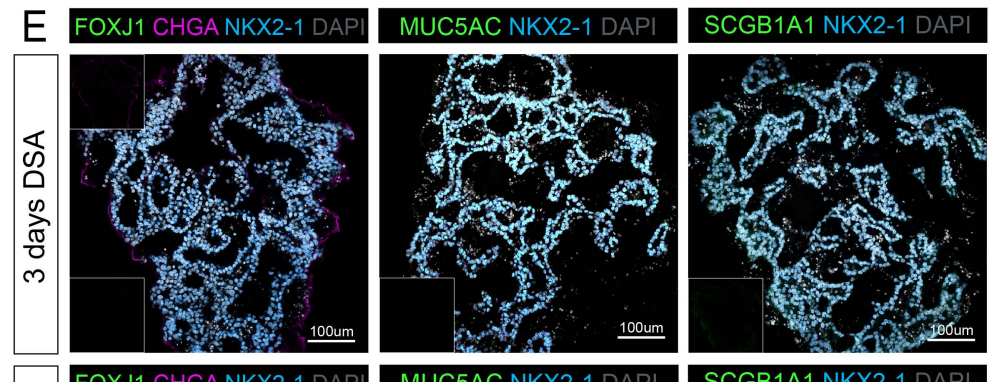

$\square$ mCherry+
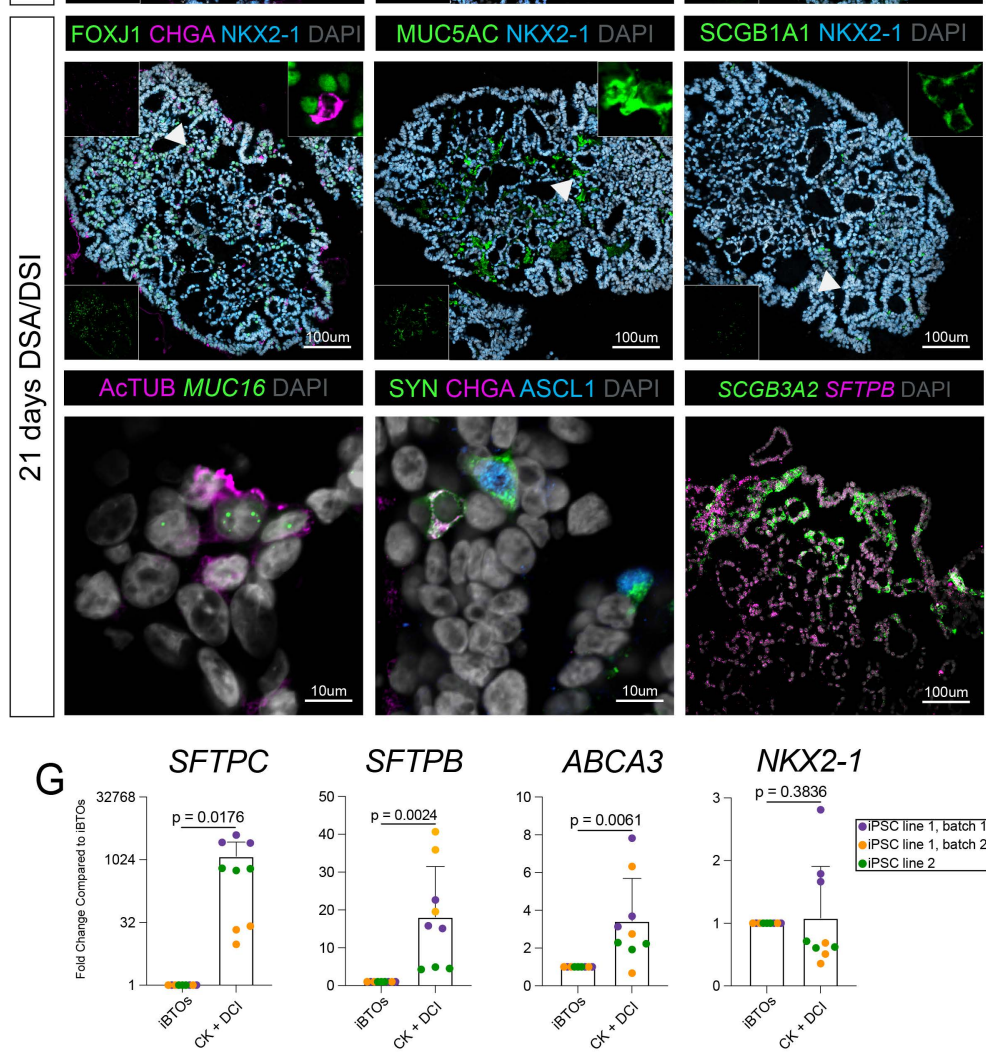

NKX2-1

3. $\mathrm{p}=0.3836$

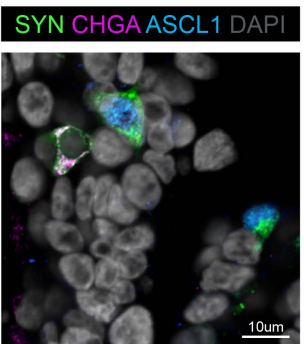

$100 \mathrm{um}$

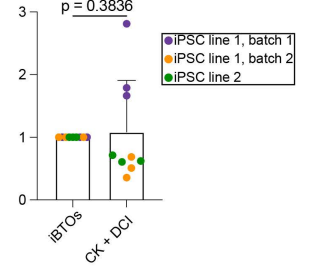




\section{Figure 4: iBTOs are Competent for Proximal Airway and Distal Alveolar Differentiation}

A) Schematic displaying the airway (Miller et al., 2020) and alveolar (Jacob et al., 2017) induction protocol from iBTOs.

B) Representative brightfield, phase contrast, and reporter expression for NKX2-1-EGFP and TP63mCherry images of iBTOs undergone the airway or alveolar induction protocol shown in Fig. 4A. Phase contrast and reporter images are from the same field.

C) Immunofluorescence staining on paraffin sections for the airway progenitor marker TP63 and lung epithelial marker NKX2-1 on iBTOs undergone 3 days of dual-SMAD activation (DSA) or 3 days DSA followed by 18 days of dual-SMAD inactivation (DSI) in the airway induction protocol.

D) FACS quantification of NKX2-1-EGFP ${ }^{+}$and mCherry-TP63+ cells from LPOs and iBTOs from the NKX2-1-EGFP/TP63-mCherry reporter cell line, either in 3F media (untreated) or after 21 days of the airway differentiation protocol (DSA/DSI treatment). Percentages of live cells expressing neither marker, each separate reporter, or dual-expressing cells are reported as mean \pm SEM for $2-3$ replicates per time point.

E) Fluorescence in situ hybridization and/or immunofluorescence staining on paraffin sections for differentiated airway epithelial markers (multiciliated: FOXJ1/AcTUB/MUC16, neuroendocrine: CHGA, SYN, ASCL1, goblet: MUC5AC, secretory: SCGB1A1, SCGB3A2, SPB) and lung epithelial marker NKX2-1 on iBTOs undergone 3 DSA or 3 days DSA followed by 18 days of DSI of the airway induction protocol. Insets on the top right are zoomed in on the area denoted by the arrows. Insets in the bottom left or top left corners are single channel images.

F) Immunofluorescence staining on paraffin sections for differentiated type II alveolar epithelial markers SPB, pro-SPC, and SPC and lung epithelial marker NKX2-1 or general epithelial marker ECAD on iBTOs and iBTOs undergone the alveolar differentiation protocol ( 7 days $\mathrm{CK}+\mathrm{DCl}$ ). Insets on the top right are zoomed in on the area denoted by the arrows. Insets in the bottom left or top left corners are single channel images.

G) RT-qPCR data comparing expression of alveolar markers SFTPC, SFTPB, and ABCA3 and lung epithelial marker NKX2-1 in untreated iBTOs (in 3F media) or after the alveolar differentiation protocol (7 days $\mathrm{CK}+\mathrm{DCl})$. Each color represents an independent experiment with a unique iPSC line or independent experiment (purple/orange: iPSC17 WT 7B2 - LPOs sorted by CPM and NKX2-1-EGFP, green: iPSC line 72.3 - LPOs sorted by CPM only). Error bars represent standard error of the mean. Statistical tests were performed by Student's t-test (paired, onetailed). 
bioRxiv preprint doi: https://doi.org/10.1101/2022.02.25.481981; this version posted February 25, 2022. The copyright holder for this preprint (which was not certified by peer review) is the author/funder, who has granted bioRxiv a license to display the preprint in perpetuity. It is made available under aCC-BY-NC-ND 4.0 International license.

\section{Supplemental Figure 1}
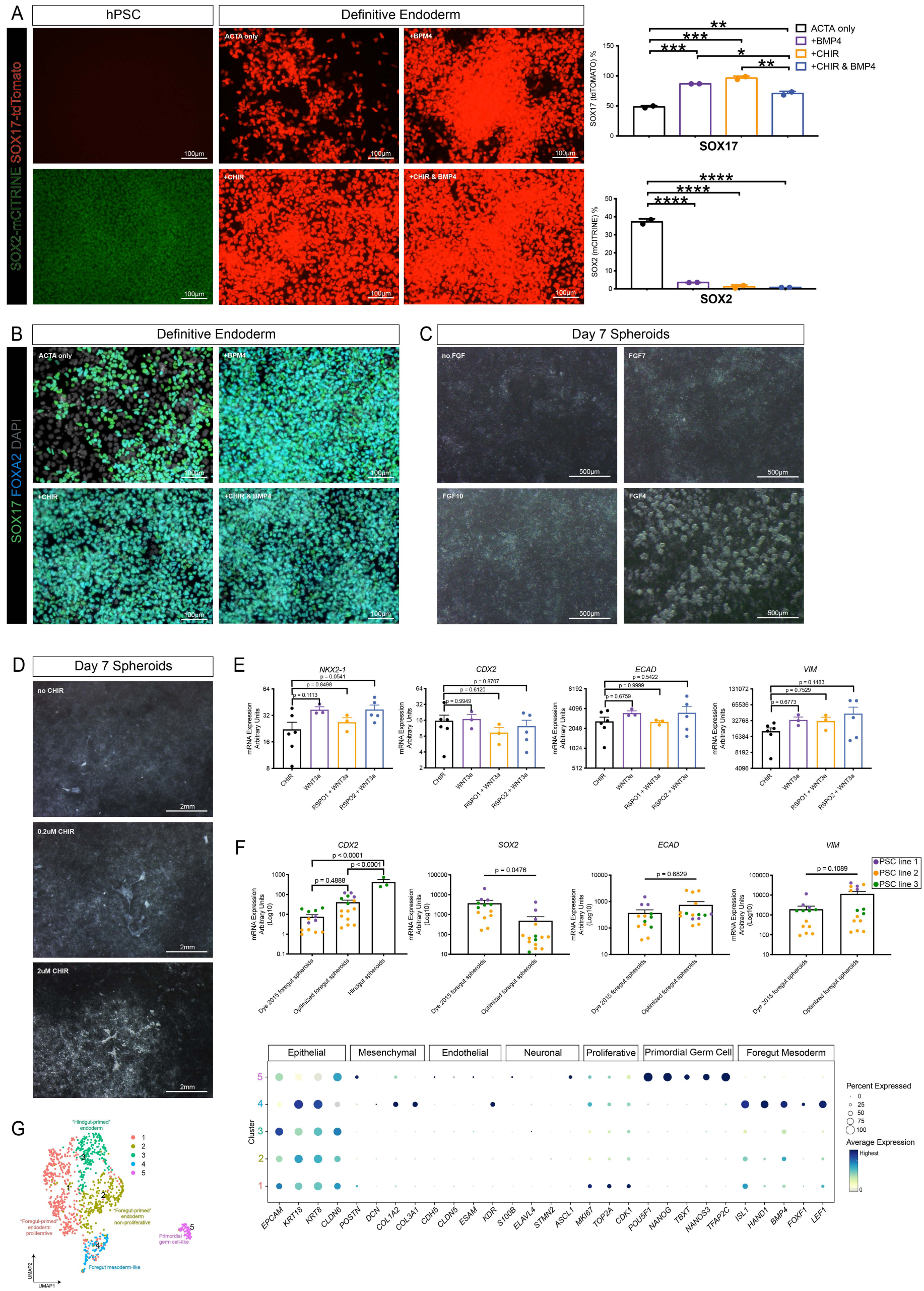


\section{Supplementary Figure 1: Optimization of Directed Differentiation of hPSCs at Definitive Endoderm,}

\section{Foregut Spheroid and NKX2-1+ Spheroid Stages}

(A) SOX2-mCitrine (pluripotent stem cell marker) and SOX17-tdTomato (definitive endoderm marker) reporter images and flow cytometry quantifications of SOX2-mCITRINE and SOX17-tdTomato on hPSCs (images only) and hPSC-derived day 4 definitive endoderm (DE) (images and flow cytometry) where $50 \mathrm{ng} / \mathrm{mL}$ BMP4 and/or $2 \mathrm{uM} \mathrm{CHIR}$ were added on day 1 of a 3-day $100 \mathrm{ng} / \mathrm{mL}$ Activin A (ACTA) treatment to induce DE (see Fig. 1A) in a hPSC line expressing SOX2mCitrine/SOX17-tdTomato hPSC line (Martyn et al., 2018). Two technical replicates from the same experiment were performed for flow cytometry quantifications. Statistical tests were performed by ordinary one-way ANOVA.

(B) Immunofluorescence staining for the DE markers SOX17 and FOXA2 on day 4 DE where $50 \mathrm{ng} / \mathrm{mL}$ BMP4 and/or 2uM CHIR were added on day 1 of a 3-day $100 \mathrm{ng} / \mathrm{mL}$ Activin A (ACTA) treatment to induce DE (see Fig. $1 A$ ). This experiment was performed on two independent $h P S C$ lines different than the reporter line in Fig. S1A. Representative images are shown from $\mathrm{H} 9$ ESCs.

(C) The requirement of FGF for the formation of spheroids on day 7 of the NKX2-1-optimized spheroid directed differentiation protocol (see Fig. 1A) was tested by adding no FGF, $10 \mathrm{ng} / \mathrm{mL}$ FGF7, 500ng/mL FGF10, or 500ng/mL FGF4 on days $4-6$.

(D) The requirement of WNT for the formation of spheroids on day 7 of the NKX2-1-optimized spheroid directed differentiation protocol was tested by adding no CHIR99021, 0.2uM CHIR99021, or 2uM CHIR99021 on days $4-6$.

(E) RT-qPCR data comparing expression of the lung epithelial marker NKX2-1, the hindgut epithelial marker $C D X 2$, the pan-epithelial marker ECAD, and the pan-mesenchymal marker VIM when CHIR99021, WNT3a, RSPO1 \& WNT3a, or RSPO2 \& WNT3a was used to activate WNT signaling on days $7-9$ of the NKX2-1-optimized spheroid directed differentiation protocol. Data points for CHIR99021 and WNT3a + RSPO2 represent 2 independent experiments with $2-3$ technical replicates and data points from other conditions represent one independent experiment with 3 technical replicates. All experiments were performed on H9 ESCs. Statistical tests compared all conditions to CHIR99021 and were performed by ordinary one-way ANOVA followed by Dunnett's multiple comparison test.

(F) RT-qPCR data comparing expression of the hindgut epithelial marker CDX2, the posterior foregut marker SOX2, the pan-epithelial marker ECAD, and pan-mesenchymal marker VIM from previously published foregut spheroids (Dye et al., 2015) to optimized foregut spheroids, and in the case of $C D X 2$, hindgut spheroids. Each color represents an independent experiment with a unique iPSC line (purple: WTC11, orange: iPSC17 WT 7B2, green: iPSC line 72.3). Each data point of the same color represents a technical replicate from the same iPSC line in one or more independent experiments. Error bars represent standard error of the mean. Statistical test for 
CDX2 was performed by ordinary one-way ANOVA followed by Turkey's multiple comparison test and statistical tests for the remaining markers was performed by a paired $t$ test.

(G) Cluster plot of scRNA-seq data from day 10 spheroids and dot plot of epithelial, mesenchymal, endothelial, and neuronal lineage genes as well as proliferation markers in each cluster of the UMAP. The dot size represents the percentage of cells expressing the gene in the corresponding cluster, and the dot color indicates log-normalized expression level of the gene. 
bioRxiv preprint doi: https://doi.org/10.1101/2022.02.25.481981; this version posted February 25, 2022. The copyright holder for this preprint (which was not certified by peer review) is the author/funder, who has granted bioRxiv a license to display the preprint in perpetuity. It is made Supplemental Figure 2

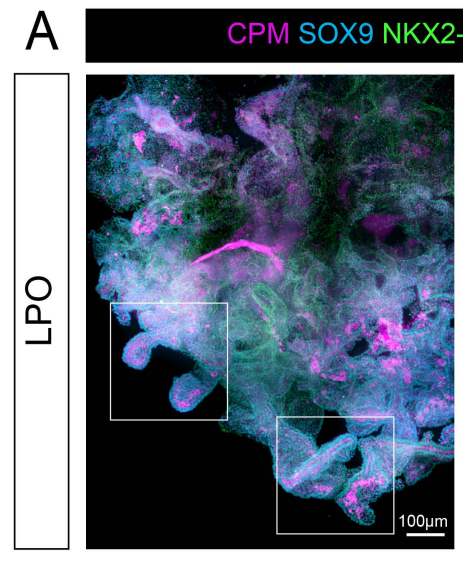

C Whole Passaged LPOs All Cell Lines

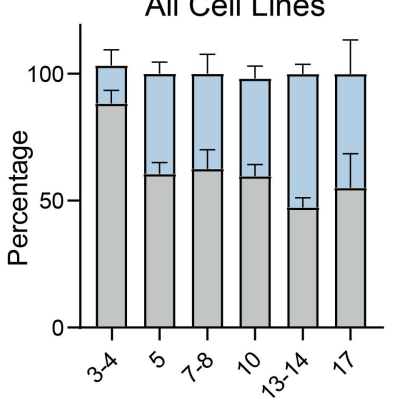

LPO age (weeks)
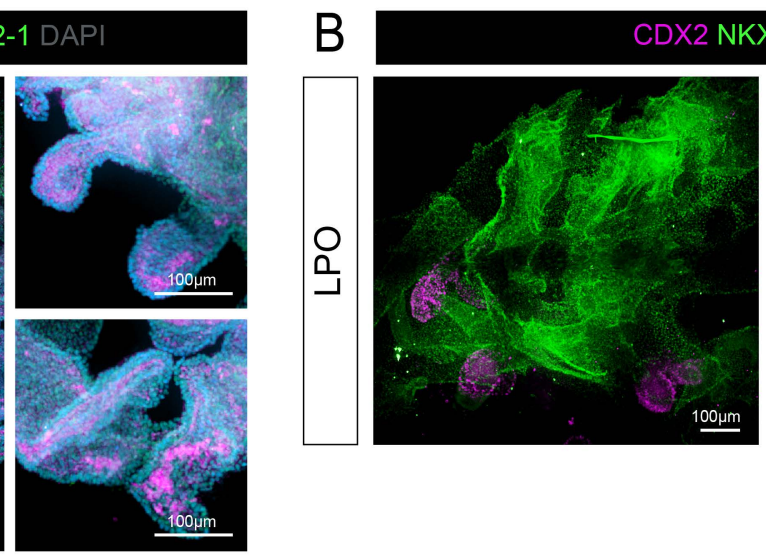

Miller et. al. 2019 Protocol

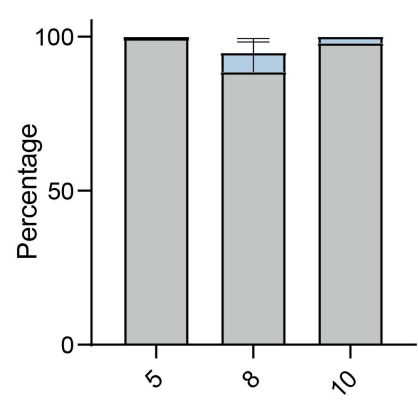

LPO age (weeks)

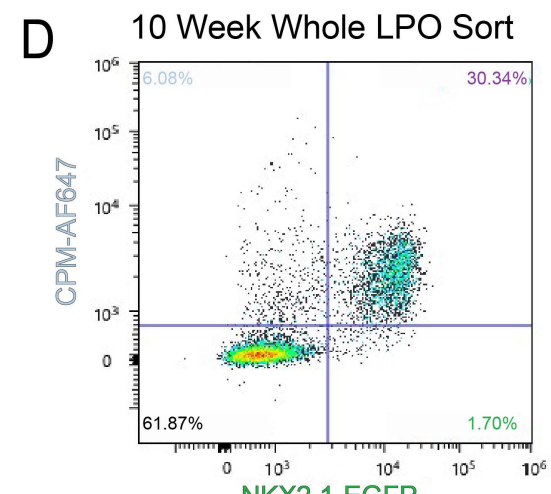

NKX2.1-EGFP
E

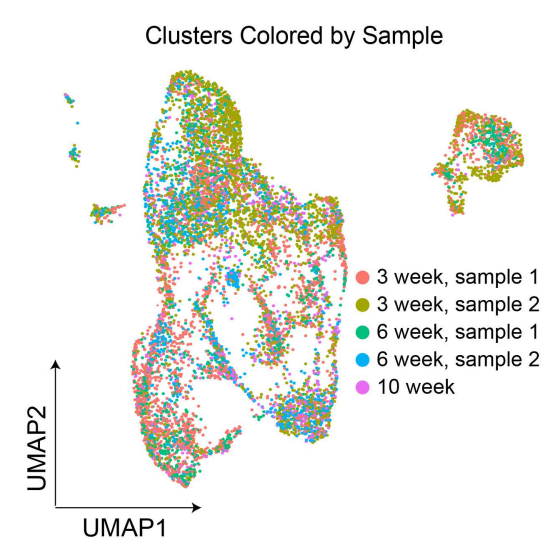

$\mathrm{F}$

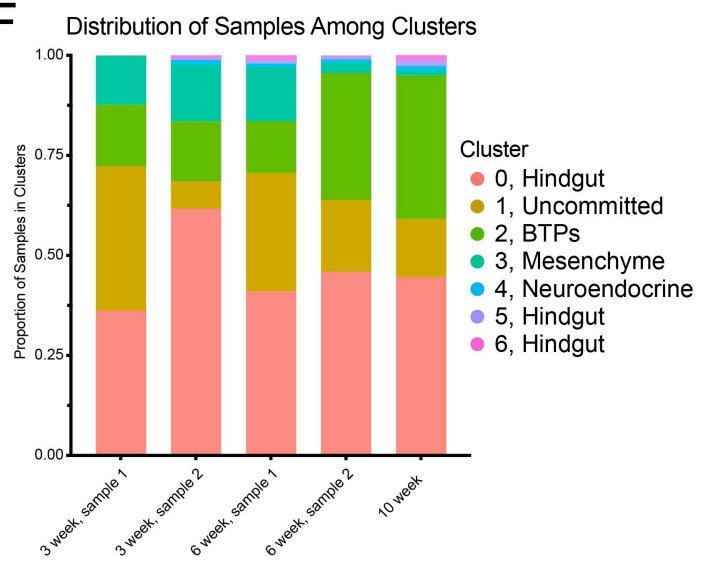

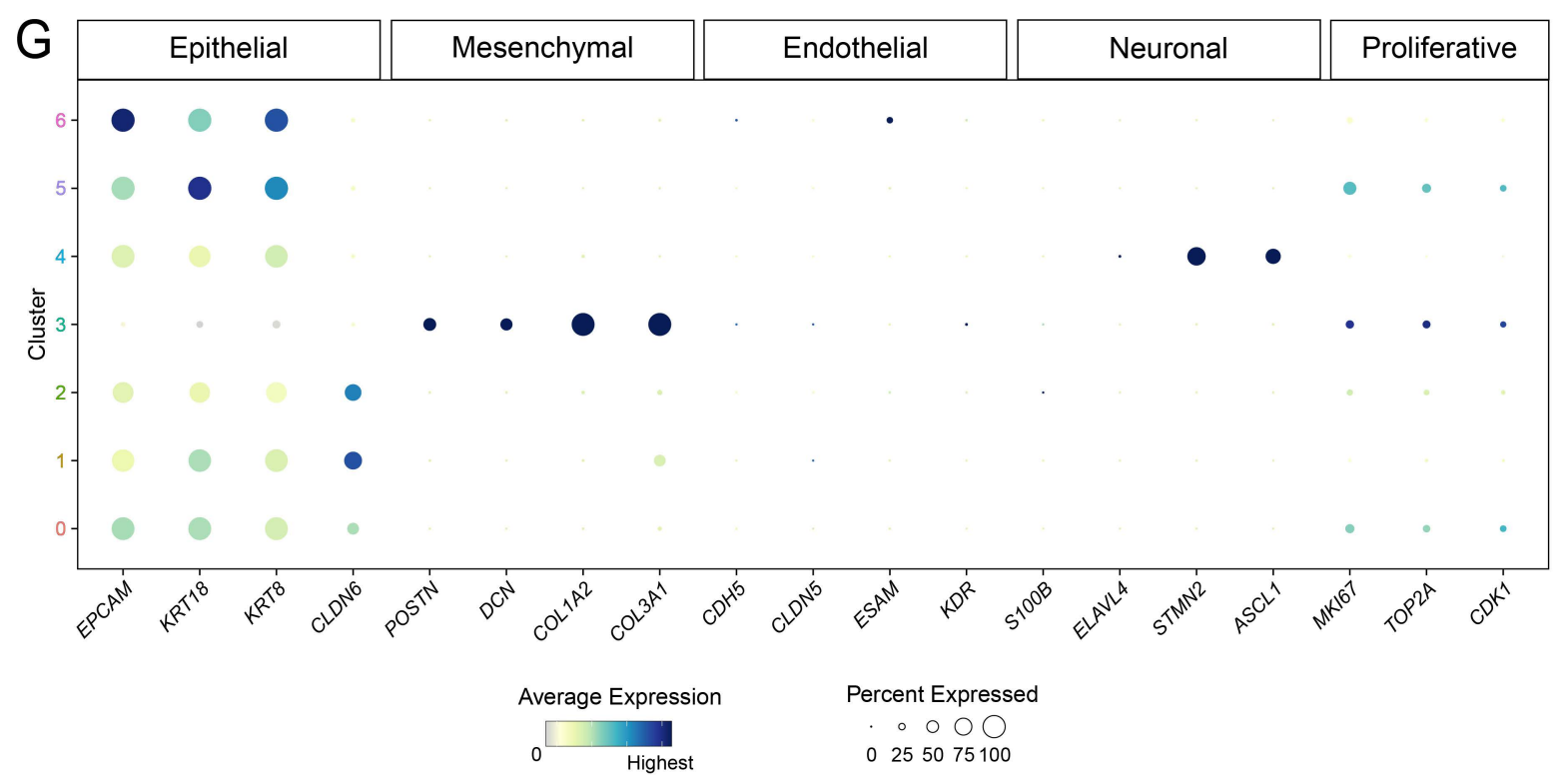




\section{Supplementary Figure 2: Bud Tip Progenitors and Contaminating Lineages Emerge and Expand in LPOs Over Time}

(A) Maximum intensity projection of a whole mount immunofluorescence confocal z-series staining of a 10-week LPO for bud tip progenitor markers CPM and SOX9 and lung epithelial marker NKX21.

(B) Maximum intensity projection of a whole mount immunofluorescence confocal z-series staining of a 10-week LPO (left) or immunofluorescence staining on paraffin sections of 4-week LPOs (right) for intestinal epithelial marker CDX2 and lung epithelial marker NKX2-1.

(C) (Left) FACS quantification of $\mathrm{CPM}^{+}$cells in LPOs in aggregate time course from three separate cell lines, including the NKX2-1-EGFP reporter line, iPSC line 72.3 and WTC11. LPOs were sorted using CPM from 3-17 weeks in 3F media. Percentages of live cells expressing CPM (blue) or negative (grey) are reported as mean \pm SEM for $2-3$ replicates per time point. (Right) FACS quantification of $\mathrm{CPM}^{+}$cells in LPOs generated from spheroids using our previous protocol from two separate cell lines, including the NKX2-1-EGFP reporter line and iPSC line 72.3 (WTC11-derived LPOs did not survive to 10 weeks) (Miller et al., 2019).

(D) Representative flow cytometry plot for iBTP selection based on positive CPM and NKX2-1-EGFP selection on 10-week LPOs.

(E) UMAP plot corresponding to the LPO cluster plot in Fig. 2G. Each dot represents a single cell and dots/cells are colored by the sample from which they came from.

(F) Stacked bar graph displaying the proportion of cells from each sample in each cluster of the LPO cluster plot in Fig. $2 \mathrm{G}$.

(G) Dot plot of epithelial, mesenchymal, endothelial, and neuronal lineage genes as well as proliferation markers in each cluster of the LPO cluster plot in Fig. 2G. The dot size represents the percentage of cells expressing the gene in the corresponding cluster, and the dot color indicates log-normalized expression level of the gene. 
bioRxiv preprint doi: https://doi.org/10.1101/2022.02.25.481981; this version posted February 25, 2022. The copyright holder for this preprint (which was not certified by peer review) is the author/funder, who has granted bioRxiv a license to display the preprint in perpetuity. It is made available under aCC-BY-NC-ND 4.0 International license.

\section{Supplemental Figure 3}

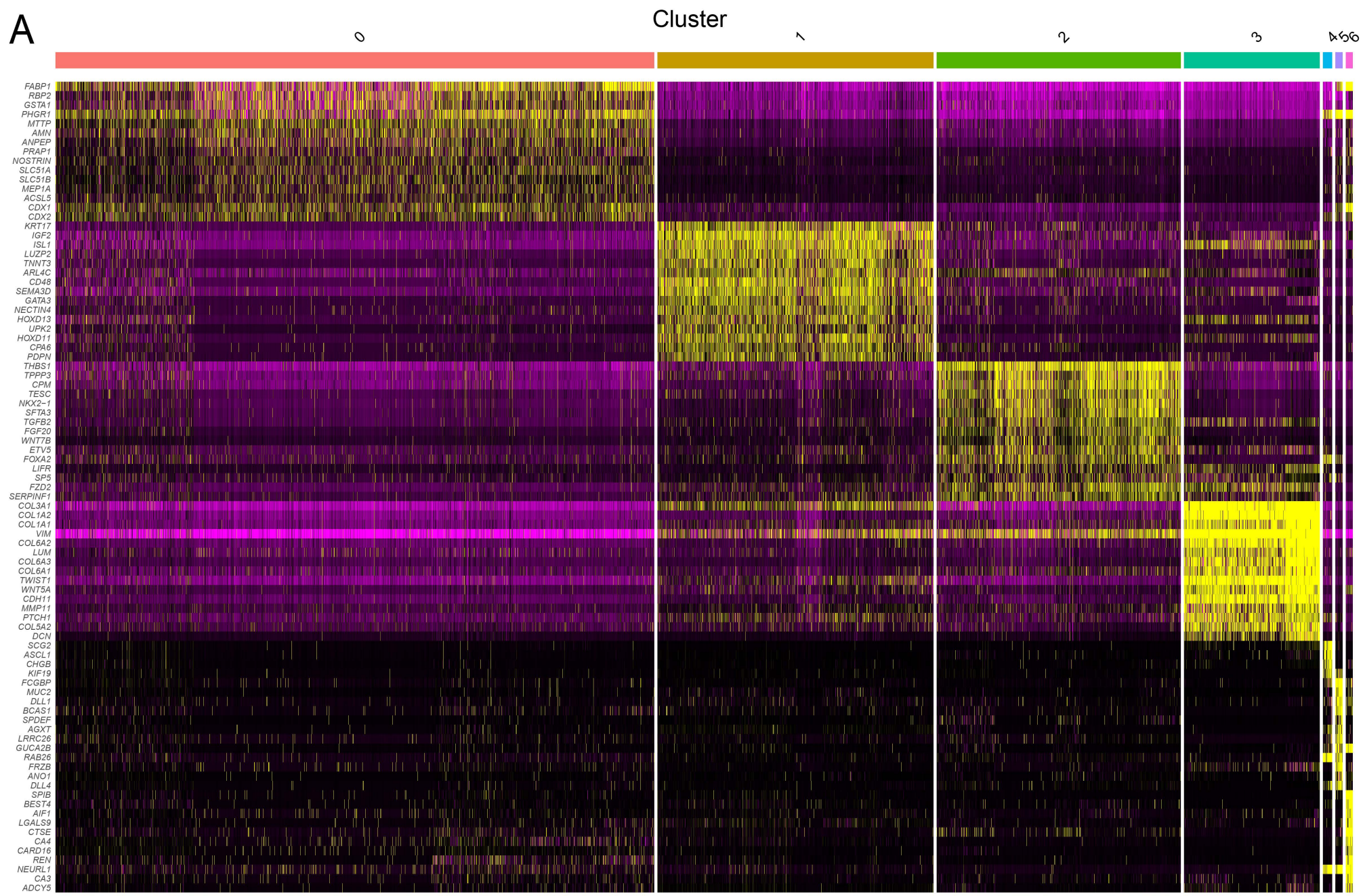


bioRxiv preprint doi: https://doi.org/10.1101/2022.02.25.481981· this version posted February 25, 2022. The copyright holder for this preprint

(which was not certified by peer review) is the author/funder, who has granted bioRxiv a license to display the preprint in perpetuity. It is made available under aCC-BY-NC-ND 4.0 International license.

\section{Supplementary Figure 3: LPOs are Transcriptionally Heterogenous}

(A) Heat map including 12 genes per cluster picked from the top-50 highly enriched genes in each cluster corresponding to the UMAP cluster plot in Fig. 2G. Top-50 genes were based on log-fold change of each gene. 
bioRxiv preprint doi: https://doi.org/10.1101/2022.02.25.481981; this version posted February 25, 2022. The copyright holder for this preprint (which was not certified by peer review) is the author/funder, who has granted bioRxiv a license to display the preprint in perpetuity. It is made available under aCC-BY-NC-ND 4.0 International license.

\section{Supplemental Figure 4}

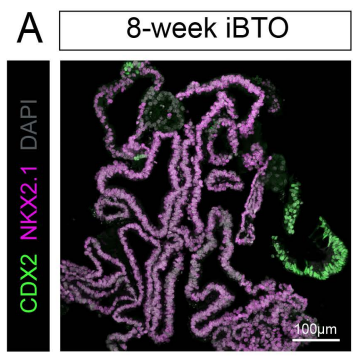

B

Mesenchymal
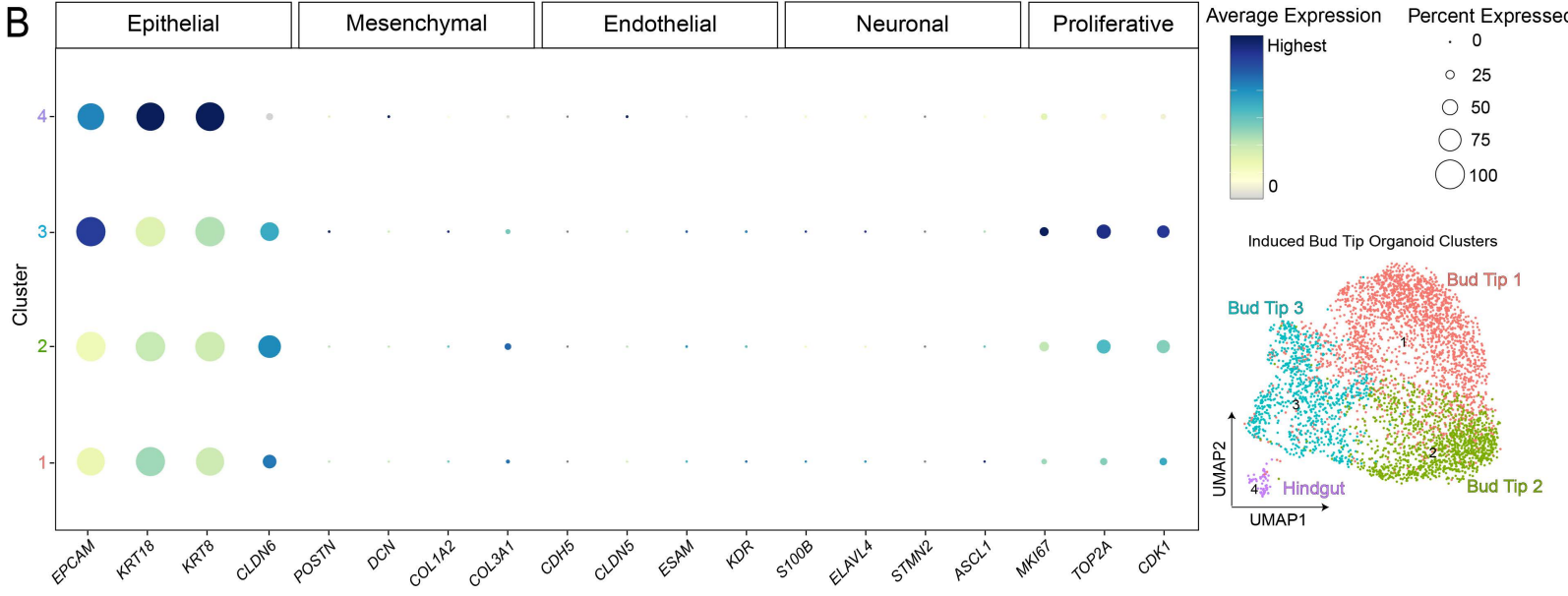

C

In Vivo Neuroendocrine Cell Score

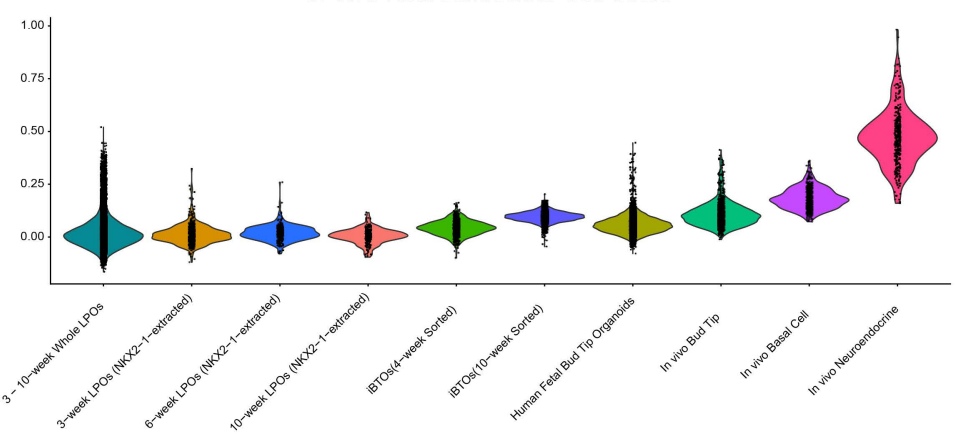

In Vivo Basal Cell Score

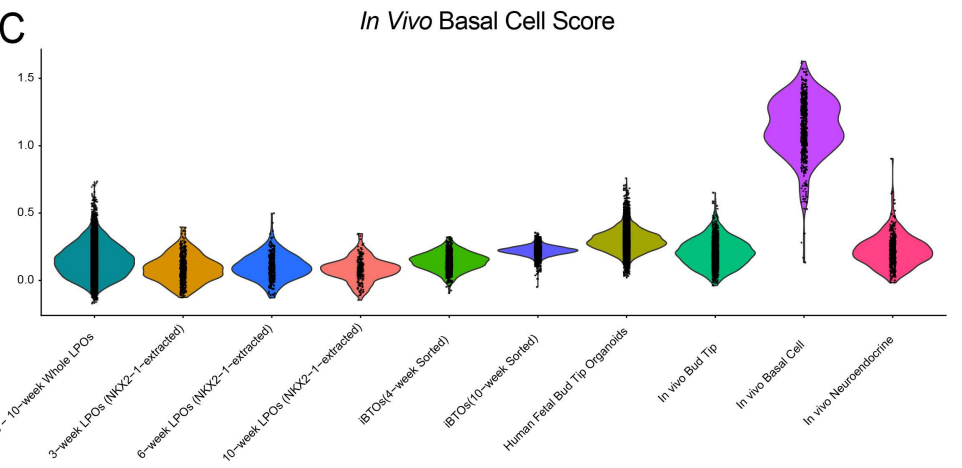




\section{Supplementary Figure 4: Induced Bud Tip Organoids are Enriched for Bud Tip Progenitor Cells}

(A) Immunofluorescence staining on paraffin sections for lung epithelial marker NKX2-1 and intestinal epithelial marker CDX2 on 8-week iBTOs.

(B) Dot plot of epithelial, mesenchymal, endothelial, and neuronal lineage genes as well as proliferation markers in each cluster of the iBTO cluster plot in Fig. 3E. The dot size represents the percentage of cells expressing the gene in the corresponding cluster, and the dot color indicates log-normalized expression level of the gene.

(C) Violin plot displaying an in vivo basal or neuroendocrine cell score, calculated as the average expression of the top 100 (basal) or 150 (neuroendocrine) enriched genes in in vivo basal or neuroendocrine cells, for each sample. Samples include whole LPOs (2x3-week LPOs, 2x6-week LPOs, 1x10-week LPOs), NXK2-1-extracted cells from 3-, 6-, and 10-week LPOs ( $n=2$ for 3- and 6-week LPOs, $n=1$ for 10-week LPOs), whole iBTOs (derived from LPOs sorted for NKX2$1^{+} / \mathrm{CPM}^{+}$cells at 4 or 10 weeks, $\mathrm{n}=1$ of each), human fetal-derived bud tip organoids, human fetal-derived bud tip organoids induced to airway (Miller et al., 2020) (airway organoids), and primary in vivo tissue including computationally-extracted bud tip, basal, and neuroendocrine cells (Miller et al., 2020; Hein et al., 2021). The cell scoring lists were generated from scRNA-seq data from the in vivo basal or neuroendocrine cells shown on the plots. 
bioRxiv preprint doi: https://doi.org/10.1101/2022.02.25.481981; this version posted February 25, 2022. The copyright holder for this preprint (which was not certified by peer review) is the author/funder, who has granted bioRxiv a license to display the preprint in perpetuity. It is made available under aCC-BY-NC-ND 4.0 International license.

\section{Supplemental Figure 5}
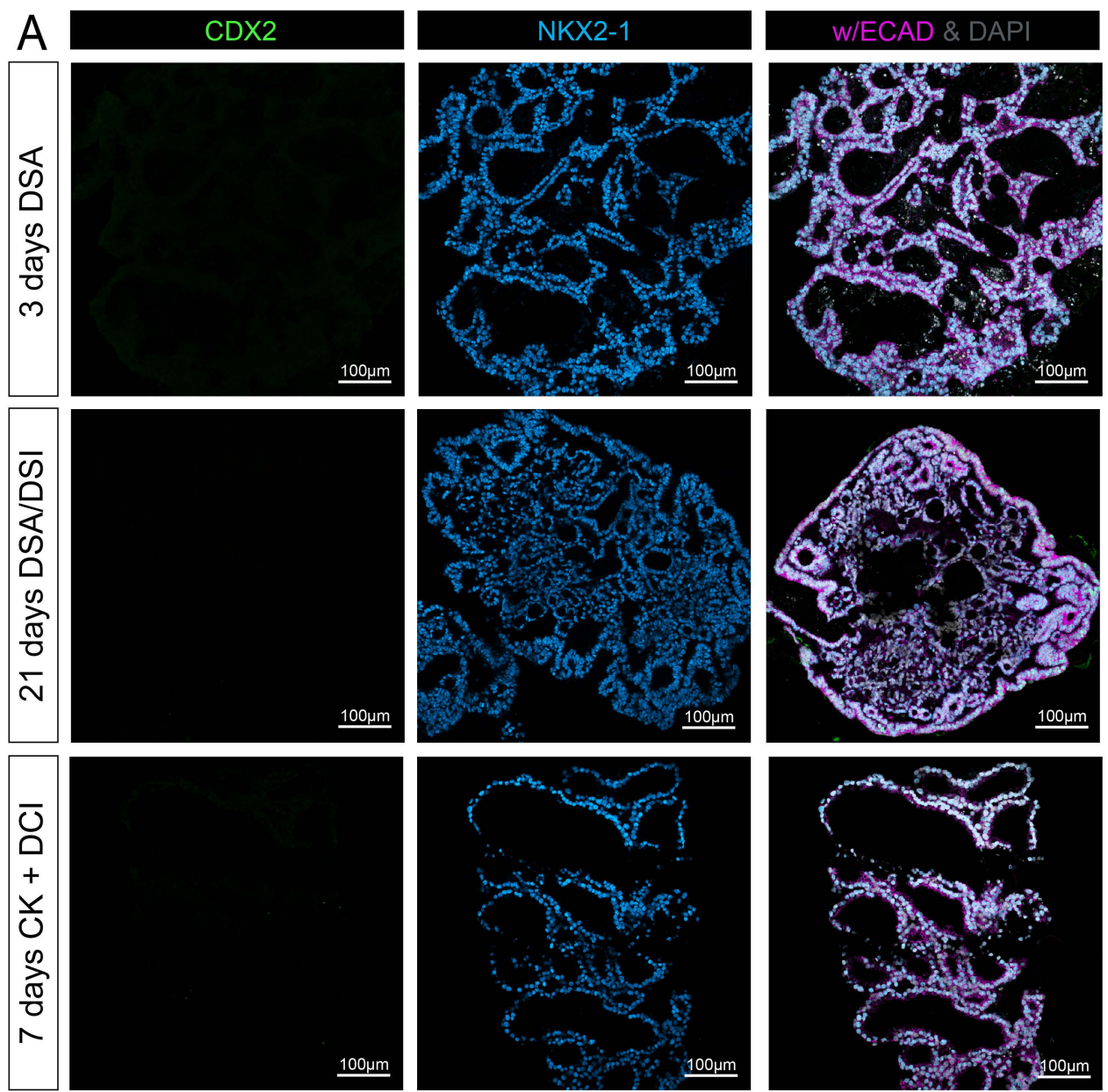

B
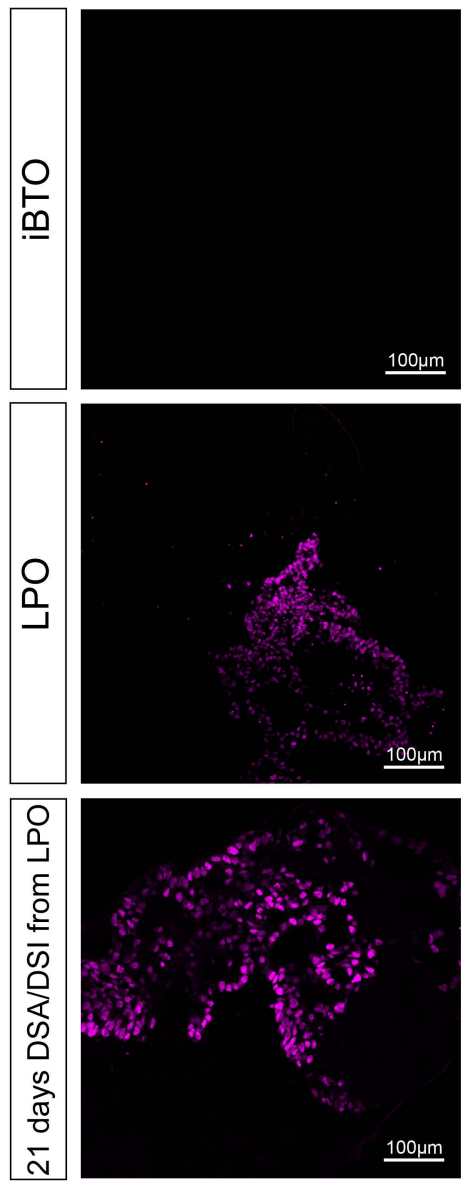
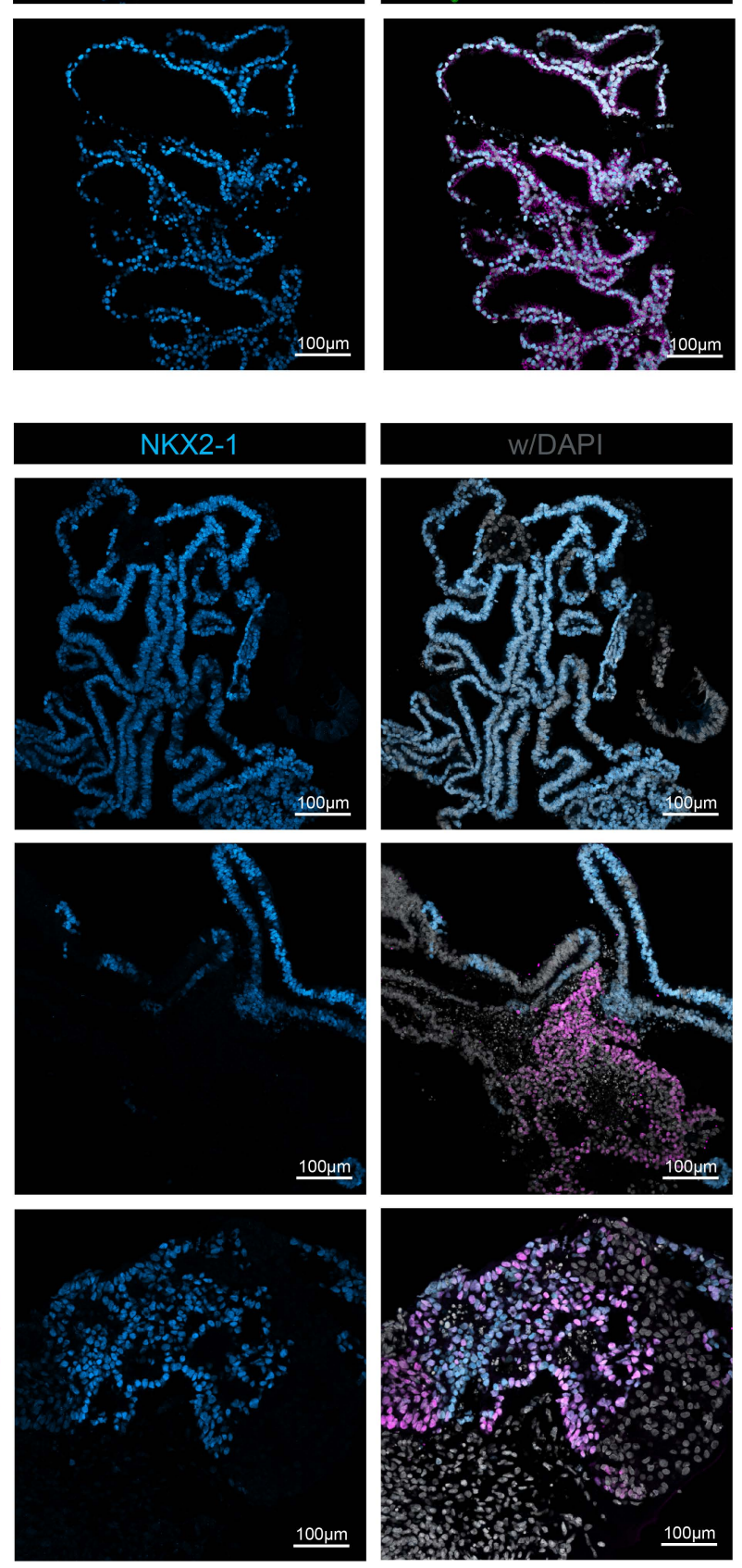


\section{Supplementary Figure 5: iBTOs Maintain NKX2-1 Expression after Differentiation into Proximal Airway and Distal Alveolar Organoids, and Differentiate More Effectively Compared to LPOs}

(A) Immunofluorescence staining on paraffin sections for the intestinal epithelial marker CDX2, lung epithelial marker NKX2-1, and general epithelial cell-type marker ECAD on 12-week iBTOs undergone 3 days of dual-SMAD activation (DSA) or 3 days DSA followed by 18 days of dualSMAD inactivation (DSI) of the airway induction protocol or 3-week iBTOs undergone the alveolar differentiation protocol (7 days $\mathrm{CK}+\mathrm{DCl}$ ) (see Fig. 4A).

(B) Immunofluorescence staining on paraffin sections for the airway progenitor marker TP63 and lung epithelial marker NKX2-1 on 12-week iBTOs, 4-week LPOs, and 3-week LPOs after 21 days of the airway differentiation protocol (DSA/DSI treatment). 